\title{
The role of string topology in symplectic field theory
}

\author{
K. Cieliebak and J. Latschev
}

5 October 2007

\section{Introduction}

Over the past two years, we have developed a program for incorporating holomorphic curves with boundary on some closed Lagrangian submanifold into the general framework of symplectic field theory (SFT). These notes give an outline of the proposed theory, with an emphasis on ideas, geometric intuition and a description of the resulting algebraic structures. Precise proofs involve a substantial amount of technical work and will appear elsewhere [9, 10].

The starting point of our work is the observation, originally due to Fukaya [18], that the new phenomena in the compactification of moduli spaces that occur for curves with boundary are usefully described in terms of the string topology of Chas and Sullivan [5]. Fukaya's work concerns the case of holomorphic disks with boundary on some closed Lagrangian submanifold in a closed symplectic manifold. He viewed these moduli spaces as ordinary or equivariant chains on the free loop space of the Lagrangian submanifold via the evaluation at the boundary. Boundary bubbling is then modelled by the loop bracket or string bracket, depending on whether or not the disks involved come with marked points on the boundary.

Our initial goal was to apply the same idea to compare the SFT invariants of a unit cotangent bundle $S^{*} Q$ to the string topology of the underlying smooth manifold $Q$. It turns out that, when suitably interpreted, the structures on the two sides are isomorphic. The simplest statement of this isomorphism is discussed in Section 7 of these notes. On the SFT side, it involves the linearized contact homology $H C^{\operatorname{lin}}\left(T^{*} Q\right)$ of the cotangent bundle $T^{*} Q$. Such a linearization in fact exists for any exact symplectic manifold with a convex end. Moreover, one can derive the following result from the algebraic properties of SFT as described in [16]:

Theorem A (cf. Theorem 6.1). Every exact symplectic manifold $(X, d \lambda)$ with convex boundary gives rise to a linearized contact homology $H^{\operatorname{lin}}(X, \lambda)$ which has the structure of an involutive graded Lie bialgebra. 
A precise definition of this concept is given in Section 5. It might be interesting to note that the bracket and cobracket are defined in terms of curves of genus 0 , but the proof of the involutivity property involves moduli spaces of curves of genus 1 . We also point out that on the chain level one has the "infinity version" of this structure. The homotopy theory of these chain-level structures is the subject of joint work with K. Fukaya [8].

In the context of Theorem $\mathrm{A}$, suppose further that $(X, d \lambda)$ contains an exact Lagrangian submanifold $Q \subset X$ which is oriented and relatively spin. Consider its string homology $H(\Sigma Q, Q)$, i.e. the singular homology (with rational coefficients) of the space $\Sigma Q=C^{0}\left(S^{1}, Q\right) / S^{1}$ of strings (= loops up to rotation in the domain) relative to the constant strings. Chas and Sullivan [6] showed that it too carries the structure of a graded involutive Lie bialgebra. Now we have

Theorem B (cf. Theorem 6.2). The moduli spaces of holomorphic disks with one puncture and boundary on $Q \subset(X, d \lambda)$ give rise to a chain map inducing a morphism of involutive Lie bialgebras

$$
H C^{\operatorname{lin}}(X, \lambda) \rightarrow H(\Sigma Q, Q) .
$$

Again we remark that one in fact expects a chain-level version of this theorem, giving a morphism of the underlying "infinity versions" of the structure. However the precise formulation of this chain level statement is complicated by the fact that the string topology operations are only partially defined, and so far there does not exist a precise statement of the invariance properties of the "full structure" they generate.

In any case, specializing to the case of cotangent bundles, i.e. $X=T^{*} Q$ and $Q \subset X$ the zero section and using the fact that the above morphism respects suitable filtrations on both sides, one obtains the following

Theorem $\mathbf{C}$ (cf. Theorem 7.1). For each closed oriented manifold Q, there is an isomorphism of involutive graded Lie bialgebras

$$
H C^{\operatorname{lin}}\left(T^{*} Q, \lambda\right) \stackrel{\cong}{\longrightarrow} H(\Sigma Q, Q) .
$$

This result should be viewed as an $S^{1}$-equivariant analogue of the isomorphism

$$
H F_{*}\left(T^{*} Q\right) \cong H_{*}(\Lambda Q)
$$

between the Floer homology of the cotangent bundle $T^{*} Q$ and the singular homology of the free loop space $\Lambda Q=\operatorname{Map}\left(S^{1}, Q\right)$, intertwining the pair-ofpants product on $H F_{*}\left(T^{*} Q\right)$ with the loop product on $H_{*}(\Lambda Q)$ (see [28], [25], [1]). Note, however, that the isomorphism of Theorem $\mathrm{C}$ is more intricate, as it respects a much more elaborate algebraic structure. At present, there is no known interesting bialgebra structure on the non-equivariant homology of the free loop space.

The string topology Lie bialgebra in the case when $Q$ is a surface is the highly non-trivial structure discovered earlier by Goldman and Turaev. In Section 7, 
we illustrate Theorem $\mathrm{C}$ by translating known properties of this Lie bialgebra into existence results for holomorphic curves with suitable asymptotics in the symplectization of $S^{*} Q$.

One of the main points of these notes is that the above results naturally fit into a more general theory of holomorphic curves with boundary on some closed Lagrangian submanifold $Q \subset X$ which is oriented and relatively spin (but not necessarily exact) in a general symplectic cobordism $X$ between contact manifolds $\left(Y^{ \pm}, \lambda^{ \pm}\right)$. The main result of this theory will be a master equation for a suitable generating function $\mathbf{L}$ for the moduli spaces of holomorphic curves with boundary on $Q$ which directly generalizes the corresponding equation for the potential $\mathbf{F}$ of the symplectic cobordism known from SFT: ${ }^{1}$

Theorem D (cf. Theorem 8.1). The potential $\mathbf{L}$ of a (not necessarily exact) pair $(X, Q)$ satisfies the master equation

$$
(\partial+\Delta+\hbar \nabla)\left(e^{\mathbf{L}}\right)=e^{\mathbf{L}} \overleftarrow{\mathbf{H}^{+}}-\overrightarrow{\mathbf{H}^{-}} e^{\mathbf{L}}
$$

where $\Delta$ and $\nabla$ are operations in string topology described in Section 4.

As we discuss in Section 8, this result can be viewed as a generalization of Fukaya's equation [18]

$$
\partial \mathbf{a}_{1}+\frac{1}{2}\left[\mathbf{a}_{1}, \mathbf{a}_{1}\right]_{\text {string }}=0
$$

for the analogous generating function $\mathbf{a}_{1}$ counting holomorphic disks with boundary on $Q \subset X$ (and without punctures). In order to use this result to construct new invariants of Lagrangian submanifolds, one needs to understand chain-level string topology. Our approach to this problem will be the subject of [10].

Theorem D also leads to a precise formulation (cf. Theorem 8.3) of the principle that "holomorphic curves with punctures asymptotic to a Lagrangian submanifold $Q$ carry the same information as holomorphic curves with boundary on Q".

One of the big open problems in the developement of SFT (besides the analytic foundations) is the formulation of its relative version. We believe that one geometrically very appealing solution to this problem can be given with the help of string topology. Our ideas in this direction, which were developed in discussions with K. Mohnke, are sketched in Appendix A.

We finish this introduction with some remarks about the structure of these notes. To bring out the main ideas more clearly, we have tried to avoid the discussion of technicalities in the main text as much as possible. At the end of each section we then list some "technical remarks" that outline more precisely the current status of the results stated and mention the main technical issues that need to be resolved in order to give complete proofs. Section 2 starts with a quick review of the algebraic formalism of SFT as described in [16].

\footnotetext{
${ }^{1}$ Strictly speaking, at the time of writing this theorem is still conjectural, cf. technical remarks in Sections 3 and 8.
} 
In Section 3 we then give a heuristic derivation of the master equation with Lagrangian boundary conditions. Section 4 makes this somewhat more precise by describing the necessary background from string topology. In Section 5 we present some algebraic concepts, and in Section 6 we discuss how these structures can be used to describe both SFT and string topology. Section 7 discusses the special case of cotangent bundles. Up to this point, we consider a simplified setup, where all symplectic cobordisms and Lagrangian submanifolds are assumed exact. In Section 8 we describe the changes needed in the general case. Finally, in Appendix A, which is joint work with K. Mohnke, we outline our approach to relative SFT.

Acknowledgements. In the course of this work, we profited in various degrees from discussions with other mathematicians. The idea of relating SFT to string topology arose at a conference in Stare Jablonki in the summer of 2004, where K.C. attended a talk of K. Fukaya and subsequently discussed the case of cylindrical contact homology with him. We also want to thank F. Bourgeois, A. Cattaneo, T. Ekholm, Y. Eliashberg, H. Hofer, D. Indelicato, K. Mohnke, K. Ono, and D. Sullivan for stimulating conversations.

\section{Symplectic field theory}

In this section we recall the Weyl formalism of SFT from [16]. Here, as in most of this paper (with the exception of Section 8), we work in the exact category, by which we mean the following:

(i) All symplectic cobordisms $(X, \omega)$ and Lagrangian submanifolds $Q \subset X$ are exact, i.e. $\omega=d \lambda$ for a 1 -form $\lambda$ restricting to a contact form on the boundary and $\left.\lambda\right|_{Q}$ is exact.

(ii) All symplectic cobordisms $(X, \omega)$ and Lagrangian submanifolds have vanishing first Chern class resp. Maslov class. Moreover, all closed Reeb orbits $\gamma: S^{1} \rightarrow Y^{ \pm}$are nondegenerate and there exist trivializations of $\gamma^{*} T X$ with respect to which all relative first Chern classes vanish.

(iii) All Lagrangian submanifolds are oriented and relatively spin.

For example, these assumptions are satisfied in the following two cases:

(1) $X=T^{*} Q$ is the cotangent bundle of an oriented manifold and $Q$ the zero section. Here condition (i) is clear for the canonical 1-form, $c_{1}\left(T^{*} X\right)=0$, and $Q$ is relatively spin. The rest of condition (ii) follows by choosing trivializations of $T\left(T^{*} Q\right)$ induced by trivializations of $T Q$ along loops.

(2) $X$ is a simply connected Stein manifold with $c_{1}=0$ and $Q$ is an oriented, spin, exact Lagrangian submanifold with vanishing Maslov class. Such manifolds arise e.g. as vanishing cycles in fibres of exact Lefschetz fibrations over the disk (in which case $Q \cong S^{n}$ ).

Contact manifolds. Let $\left(Y^{2 n-1}, \lambda\right)$ be a closed contact manifold. Use the trivializations provided by the exactness hypothesis to define the ConleyZehnder index $\mathrm{CZ}(\gamma) \in \mathbb{Z}$ of a closed Reeb orbit $\gamma: S^{1} \rightarrow Y$. Recall that the 
$k$-th iterate $\bar{\gamma}^{k}$ of a simple closed Reeb orbit $\bar{\gamma}$ is called good if $\mathrm{CZ}\left(\bar{\gamma}^{k}\right) \equiv \mathrm{CZ}(\bar{\gamma})$ mod 2. To each good closed Reeb orbit $\gamma$ associate formal variables $p_{\gamma}, q_{\gamma}$ with gradings

$$
\left|p_{\gamma}\right|:=n-3-\mathrm{CZ}(\gamma), \quad\left|q_{\gamma}\right|:=n-3+\mathrm{CZ}(\gamma) .
$$

We denote by $\kappa_{\gamma}$ the multiplicity of the orbit $\gamma$. Let $J$ be a cylindrical almost complex structure on $\mathbb{R} \times V$ adjusted to $\lambda$. For ordered collections of closed Reeb orbits $\Gamma^{ \pm}=\left(\gamma_{1}^{ \pm}, \ldots, \gamma_{s^{ \pm}}^{ \pm}\right)$and an integer $g \geq 0$ denote by

$$
\mathcal{M}_{g}\left(\Gamma^{-}, \Gamma^{+}\right)
$$

the moduli space of connected $J$-holomorphic curves of genus $g$ in $\mathbb{R} \times Y$ with $s^{+}$positive and $s^{-}$negative punctures asymptotic to the $\gamma_{i}^{+}$resp. $\gamma_{j}^{-}$. The exactness hypothesis ensures that the following dimension formula holds:

$$
\operatorname{dim} \mathcal{M}_{g}\left(\Gamma^{-}, \Gamma^{+}\right)=(n-3)\left(2-2 g-s^{+}-s^{-}\right)+\sum_{i} \mathrm{CZ}\left(\gamma_{i}^{+}\right)-\sum_{j} \mathrm{CZ}\left(\gamma_{j}^{-}\right) .
$$

If this dimension is 1 denote by $n_{g}\left(\Gamma^{-}, \Gamma^{+}\right) \in \mathbb{Q}$ the algebraic count of points in $\mathcal{M}_{g}\left(\Gamma^{-}, \Gamma^{+}\right) / \mathbb{R}$. We introduce the formal variables

$$
p:=\sum_{\gamma} \frac{1}{\kappa_{\gamma}} p_{\gamma} \gamma, \quad q:=\sum_{\gamma} \frac{1}{\kappa_{\gamma}} q_{\gamma} \gamma
$$

and the correlator

$$
{ }^{-1}\langle\underbrace{q, \ldots, q}_{s^{-}} ; \underbrace{p, \ldots, p}_{s^{+}}\rangle_{g}:=\sum_{\left|\Gamma^{ \pm}\right|=s^{ \pm}} n_{g}\left(\Gamma^{-}, \Gamma^{+}\right) q^{\Gamma^{-}} p^{\Gamma^{+}},
$$

and set

$$
\mathbf{H}_{g}:=\sum_{s^{-}, s^{+}} \frac{1}{s^{-} ! s^{+} !}{ }^{-1}\langle\underbrace{q, \ldots, q}_{s^{-}} ; \underbrace{p, \ldots, p}_{s^{+}}\rangle_{g} .
$$

Finally, let $\hbar$ be another formal variable of degree

$$
|\hbar|=2(n-3)
$$

and define the Hamiltonian

$$
\mathbf{H}:=\frac{1}{\hbar} \sum_{g=0}^{\infty} \mathbf{H}_{g} \hbar^{g} .
$$

The grading conventions are such that $\mathbf{H}$ is homogeneous of degree -1 .

Let $\mathcal{W}$ be the graded Weyl algebra of power series in the variables $\hbar, p_{\gamma}$ with coefficients polynomial in the variables $q_{\gamma} . \mathcal{W}$ is equipped with the associative product $*$ in which all variables super-commute according to their gradings except for the variables $p_{\gamma}, q_{\gamma}$ corresponding to the same Reeb orbit $\gamma$, for which we have

$$
p_{\gamma} * q_{\gamma}-(-1)^{\left|p_{\gamma}\right|\left|q_{\gamma}\right|} q_{\gamma} * p_{\gamma}=\kappa_{\gamma} \hbar .
$$


Let $\mathcal{P}$ be the graded commutative algebra of power series in the variables $p_{\gamma}$ with coefficients polynomial in the variables $q_{\gamma}$. Using the commutation relations, every element $F \in \mathcal{W}$ can be uniquely written in the standard form

$$
F=\sum_{\Gamma, g} f_{\Gamma, g}(q) p^{\Gamma} \hbar^{g}
$$

with polynomials $f_{\Gamma, g}$. This gives us an identification $\mathcal{W} \cong \mathcal{P}[[\hbar]]$ as vector spaces. From now on we will use this identification, so all elements of $\mathcal{W}$ are assumed to be written in the standard form (3).

The following theorem encodes the boundaries of 2-dimensional moduli spaces via gluing of holomorphic curves, see Figure 1.

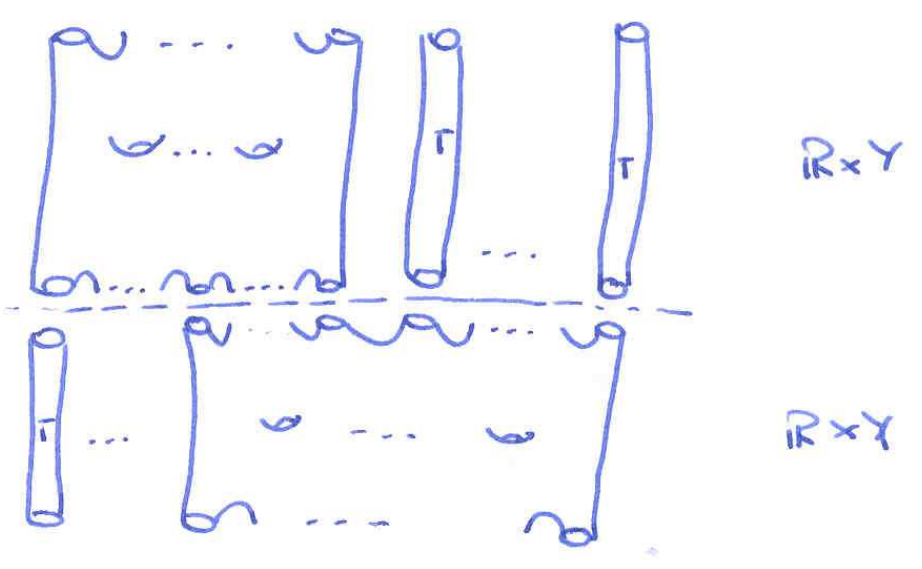

Figure 1: Breaking of curves in a symplectization

Theorem 2.1 ([16]). The Hamiltonian $\mathbf{H} \in \frac{1}{\hbar} \mathcal{W}$ satisfies the master equation

$$
\mathbf{H} * \mathbf{H}=0 .
$$

Symplectic cobordisms. Next let $\left(X^{2 n}, \omega\right)$ be an exact symplectic cobordism with convex end $\left(\mathbb{R}_{+} \times Y^{+}, \lambda^{+}\right)$and concave end $\left(\mathbb{R}_{-} \times Y^{-}, \lambda^{-}\right)$. Let $J$ be a compatible almost complex structure on $X$ that is cylindrical and adjusted to $\lambda^{ \pm}$on the ends. For ordered collections of closed Reeb orbits $\Gamma^{ \pm}=\left(\gamma_{1}^{ \pm}, \ldots, \gamma_{s^{ \pm}}^{ \pm}\right)$ in $Y^{ \pm}$and an integer $g \geq 0$ denote by

$$
\mathcal{M}_{g}\left(X ; \Gamma^{-}, \Gamma^{+}\right)
$$

the moduli space of connected $J$-holomorphic curves of genus $g$ in $X$ with $s^{+}$ positive and $s^{-}$negative punctures asymptotic to the $\gamma_{i}^{+}$resp. $\gamma_{j}^{-}$. Under our 
exactness hypothesis, this moduli space has expected dimension

$$
\operatorname{dim} \mathcal{M}_{g}\left(X ; \Gamma^{-}, \Gamma^{+}\right)=(n-3)\left(2-2 g-s^{+}-s^{-}\right)+\sum_{i} \mathrm{CZ}\left(\gamma_{i}^{+}\right)-\sum_{j} \mathrm{CZ}\left(\gamma_{j}^{-}\right) .
$$

If this dimension is 0 denote by $n_{g}\left(X ; \Gamma^{-}, \Gamma^{+}\right) \in \mathbb{Q}$ the algebraic count of points in $\mathcal{M}_{g}\left(X ; \Gamma^{-}, \Gamma^{+}\right)$. Define the correlators

$$
{ }^{0}\langle\underbrace{q, \ldots, q}_{s^{-}} ; \underbrace{p, \ldots, p}_{s^{+}}\rangle_{g}:=\sum_{\left|\Gamma^{ \pm}\right|=s^{ \pm}} n_{g}\left(X ; \Gamma^{-}, \Gamma^{+}\right) q^{\Gamma^{-}} p^{\Gamma^{+}}
$$

where the sum is taken over all ordered collections $\Gamma^{ \pm}$. Define the potential $\mathbf{F}$ of the cobordism $X$ by

$$
\mathbf{F}:=\frac{1}{\hbar} \sum_{g=0}^{\infty} \mathbf{F}_{g} \hbar^{g}
$$

where

$$
\mathbf{F}_{g}:=\sum_{s^{-}, s^{+}} \frac{1}{s^{-!} s^{+!}}{ }^{0}\langle\underbrace{q, \ldots, q}_{s^{-}} ; \underbrace{p, \ldots, p}_{s^{+}}\rangle_{g} .
$$

Denote by $\mathcal{D}$ the space of formal power series in $\hbar$ and the $p_{\gamma}^{+}$with coefficients polynomial in the $q_{\gamma}^{-}$. Elements in $\mathcal{W}^{ \pm}$act as differential operators from the right/left on $\mathcal{D}$ via the replacements

$$
q_{\gamma}^{+} \mapsto \kappa_{\gamma} \hbar \frac{\overleftarrow{\partial}}{\partial p_{\gamma}^{+}}, \quad p_{\gamma}^{-} \mapsto \kappa_{\gamma} \hbar \frac{\vec{\partial}}{\partial q_{\gamma}^{-}}
$$

The following theorem encodes the boundaries of 1-dimensional moduli spaces via gluing of holomorphic curves, see Figure 2.

Theorem 2.2 ([16]). The potential $\mathbf{F} \in \frac{1}{\hbar} \mathcal{D}$ satisfies the master equation

$$
e^{\mathbf{F}} \overleftarrow{\mathbf{H}^{+}}-\overrightarrow{\mathbf{H}^{-}} e^{\mathbf{F}}=0
$$

Higher dimensional moduli spaces. So far we have considered only rigid holomorphic curves. The generalization to higher-dimensional moduli spaces is straightforward as follows, see [17] for details. We begin again with a contact manifold $(Y, \lambda)$. A punctured $J$-holomorphic curve $f: \dot{S} \rightarrow \mathbb{R} \times Y$ asymptotic to $\Gamma^{ \pm}$induces a continuous map $\bar{f}: \bar{S} \rightarrow Y$ from the compactification $\bar{S}$ of $\dot{S}$ (replacing each puncture by a boundary circle) to $Y$ with boundary on $\Gamma^{ \pm}$. Via the assignment $f \mapsto \bar{f}$ we view a moduli space $\mathcal{M}_{g}\left(\Gamma^{-}, \Gamma^{+}\right) / \mathbb{R}$ of dimension $d$ as a $d$-chain $c_{g}\left(\Gamma^{-}, \Gamma^{+}\right)$in the space of continuous maps with boundary on $\Gamma^{ \pm}$. Define correlators

$$
\langle\underbrace{q, \ldots, q}_{s^{-}} ; \underbrace{p, \ldots, p}_{s^{+}}\rangle_{g}^{Y}:=\sum_{\left|\Gamma^{ \pm}\right|=s^{ \pm}} c_{g}\left(\Gamma^{-}, \Gamma^{+}\right) q^{\Gamma^{-}} p^{\Gamma^{+}},
$$



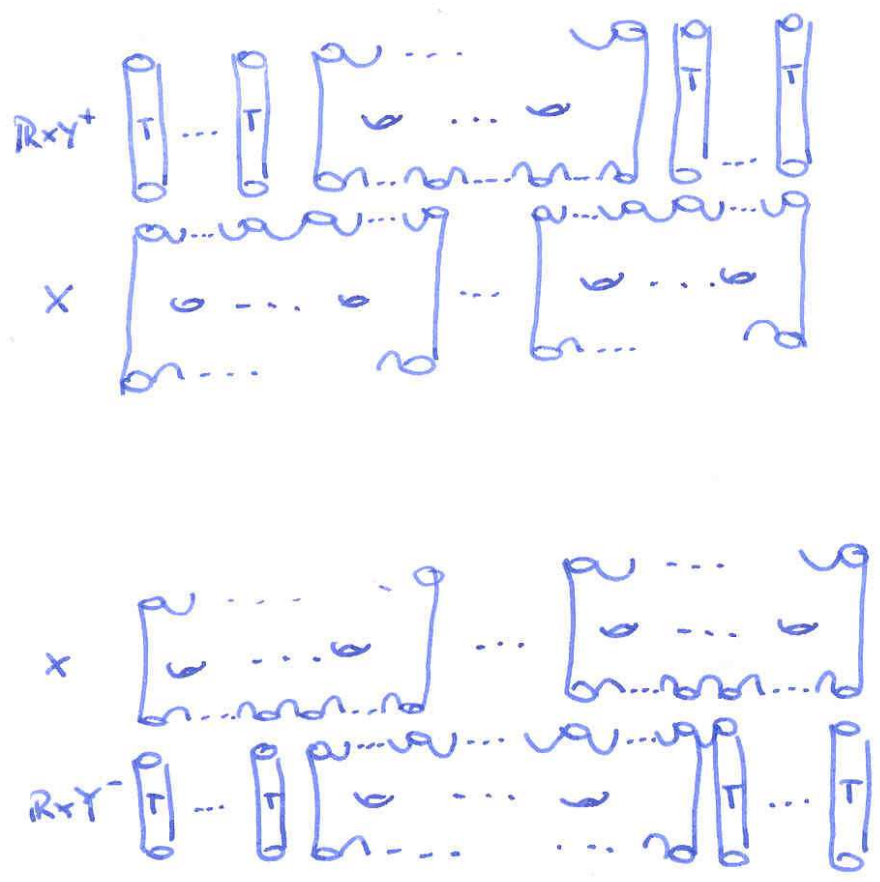

Figure 2: Breaking of curves in a cobordism

and the Hamiltonian encoding moduli spaces of all dimensions

$$
\mathbb{H}:=\frac{1}{\hbar} \sum_{g, s^{-}, s^{+}} \frac{1}{s^{-} ! s^{+}}\langle\underbrace{q, \ldots, q}_{s^{-}} ; \underbrace{p, \ldots, p}_{s^{+}}\rangle_{g}^{Y} \hbar^{g} .
$$

Then $\mathbb{H} \in \frac{1}{\hbar} \mathbb{W}$, where $\mathbb{W}$ consists of power series in $\hbar, p$, polynomial in $q$, with coefficients being chains in suitable spaces of continuous maps to $Y$. $\mathbb{W}$ is equipped with the associative product $*$ combining the $*$ product on $\mathcal{W}$ with the obvious gluing of continuous maps. Moreover, the boundary operator $\partial$ on chains acts on $\mathbb{W}$. Now Figure 1 shows that $\mathbb{H}$ satisfies the master equation

$$
\partial \mathbb{H}+\frac{1}{2} \mathbb{H} * \mathbb{H}=0 .
$$

Similarly, for a symplectic cobordism $(X, \omega)$ denote by $\mathbb{F}$ the potential encoding moduli spaces of all dimensions. Figure 2 shows that $\mathbb{F}$ satisfies the master equation

$$
\partial e^{\mathbb{F}}=e^{\mathbb{F}} \overleftarrow{\mathbb{H}^{+}}-\overleftrightarrow{\mathbb{H}^{-}} e^{\mathbb{F}}
$$




\section{Technical remarks}

The proofs of Theorems 2.1 and 2.2 require a substantial amount of technical work which is still not completed. The necessary compactness results are proven in [2] (see also [11]), and coherent orientations for the moduli spaces are discussed in [3]. What remains to be proven are the transversality and gluing results needed to describe the structure of the moduli spaces. The best one can expect is that $\mathcal{M}_{g}\left(\Gamma^{+}, \Gamma^{-}\right)$will be weighted branched manifolds in the sense of [13] with boundary and corners. The precise formulation of the structure theorems is the subject of ongoing work of Hofer, Wysocki and Zehnder [22], who introduce the machinery of polyfolds as a tool to deal with the occuring analytic difficulties.

\section{The master equation with Lagrangian bound- ary conditions}

In this section we show how degenerations of moduli spaces of punctured holomorphic curves with Lagrangian boundary conditions lead to a master equation similar to equation (7). The discussion in this section is rather informal, the definitions being postponed to Section 4.

As in the previous section, let $\left(X^{2 n}, \omega\right)$ be an exact symplectic cobordism between contact manifolds $\left(Y^{ \pm}, \lambda^{ \pm}\right)$with a compatible almost complex structure $J$. In addition, let $Q \subset X$ be an exact compact oriented Lagrangian submanifold as described at the beginning of Section 2 .

Fix ordered collections of closed Reeb orbits $\Gamma^{ \pm}=\left(\gamma_{1}^{ \pm}, \ldots, \gamma_{s^{ \pm}}^{ \pm}\right)$and integers $g, k \geq 0$. Denote by

$$
\mathcal{M}_{g, k}\left(X, Q ; \Gamma^{-}, \Gamma^{+}\right)
$$

the moduli space of connected $J$-holomorphic curves of genus $g$ in $X$ with $s^{+}$ positive and $s^{-}$negative punctures asymptotic to the $\gamma_{i}^{+}$resp. $\gamma_{j}^{-}$and with $k$ ordered boundary components on $Q$. In view of the exactness hypotheses, this moduli space has expected dimension

$$
\begin{aligned}
& \operatorname{dim} \mathcal{M}_{g, k}\left(X, Q ; \Gamma^{-}, \Gamma^{+}\right) \\
& \quad=(n-3)\left(2-2 g-s^{+}-s^{-}-k\right)+\sum_{i} \mathrm{CZ}\left(\gamma_{i}^{+}\right)-\sum_{j} \mathrm{CZ}\left(\gamma_{j}^{-}\right) .
\end{aligned}
$$

Simultaneous evaluation at the $k$ boundary circles defines a map

$$
c_{g, k}\left(\Gamma^{-}, \Gamma^{+}\right): \mathcal{M}_{g, k}\left(X, Q ; \Gamma^{-}, \Gamma^{+}\right) \rightarrow \underbrace{\sum \times \ldots \times \Sigma}_{k}=: \Sigma^{k}
$$

to the $k$-fold product of the space $\Sigma=\Sigma Q=C^{0}\left(S^{1}, Q\right) / S^{1}$ of closed strings $(=$ loops up to rotation in the domain) on $Q$. We may thus view $c_{g, k}\left(\Gamma^{-}, \Gamma^{+}\right)$as a chain in $\Sigma^{k}$. While this chain is the obvious geometric object to work with, for various technical reasons it is more convenient to consider it as a chain relative 
to the constant strings. Indeed, denote by $Q \subset \Sigma Q$ the image of the embedding that assigns to each point the constant string at that point, and set

$$
\text { const }_{k}:=\bigcup_{i=1}^{k} \Sigma \times \ldots \times \stackrel{i}{Q} \times \ldots \times \Sigma \subset \Sigma^{k} .
$$

In these notes we view $c_{g, k}\left(\Gamma^{-}, \Gamma^{+}\right)$as an element of $C_{*}\left(\Sigma^{k}\right.$, const $\left._{k}\right)$.

Define the correlators

$$
\langle\underbrace{q, \ldots, q}_{s^{-}} ; \underbrace{p, \ldots, p}_{s^{+}}\rangle_{g, k}^{X, Q}:=\sum_{\left|\Gamma^{ \pm}\right|=s^{ \pm}} c_{g, k}\left(\Gamma^{-}, \Gamma^{+}\right) q^{\Gamma^{-}} p^{\Gamma^{+}},
$$

where the sum is taken over all ordered collections $\Gamma^{ \pm}$. Define the potential $\mathbf{L}$ of the pair $(X, Q)$ by

$$
\mathbf{L}:=\frac{1}{\hbar} \sum_{g=0}^{\infty} \mathbf{L}_{g} \hbar^{g},
$$

where

$$
\mathbf{L}_{g}:=\sum_{s^{-}, s^{+}, k} \frac{1}{s^{-} ! s^{+} ! k !}\langle\underbrace{q, \ldots, q}_{s^{-}} ; \underbrace{p, \ldots, p}_{s^{+}}\rangle_{g, k}^{X, Q} .
$$

Thus $\mathbf{L}$ is a formal power series in $\hbar$ and the $p_{\gamma}^{+}$, polynomial in the $q_{\gamma}^{-}$, with coefficients in the singular chains on $\Sigma^{k}$ (where $k$ ranges over all nonnegative integers). Note that restricting to the terms of $\mathbf{L}$ corresponding to $k=0$ we recover the usual potential $\mathbf{F}$ of the cobordism as discussed in Section 2.

The following theorem encodes the codimension 1 boundaries of moduli spaces (of any dimension) of punctured holomorphic curves with boundary on $Q$. We state it here informally; the precise definition of the operations $\Delta$ and $\nabla$ will be given in Section 4.

Theorem 3.1. The potential $\mathbf{L}$ satisfies the master equation

$$
(\partial+\Delta+\hbar \nabla) e^{\mathbf{L}}=e^{\mathbf{L}} \overleftarrow{\mathbf{H}^{+}}-\overrightarrow{\mathbf{H}^{-}} e^{\mathbf{L}},
$$

where $\partial$ is the singular boundary operator and $\Delta$ resp. $\nabla$ denote the operations of decomposing one string at a self-intersection resp. gluing two strings at an intersection.

Sketch of proof. Figure 3 shows the three new phenomena in the codimension one boundary of a moduli space appearing in $\mathbf{L}$ (where for simplicity we omitted negative punctures) which are due to the Lagrangian boundary conditions. The first one is the pinching of an arc connecting two boundary loops. The inverse procedure is decomposition of a boundary loop at a self-intersection, which is encoded by the operation $\Delta$. The second boundary phenomenon is the pinching of an arc connecting one boundary loop to itself. Here the inverse procedure is gluing two boundary loops at an intersection, which is encoded by the operation 

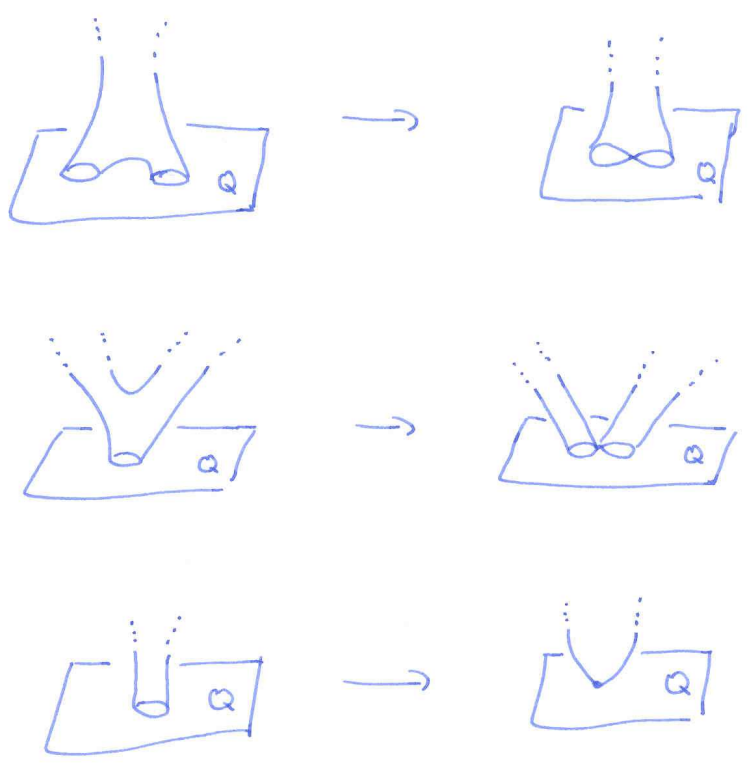

Figure 3: Codimension 1 phenomena near the boundary

$\nabla$. The third one is the shrinking of a boundary loop to a point, which leads to a chain with image in const $t_{k} \subset \Sigma^{k}$. This part of the boundary is set to zero by working with relative chains.

We point out that, as an abstract manifold, a moduli space in $\mathbf{L}$ has other codimension 1 boundary components, e.g. breaking off of higher dimensional moduli spaces in a symplectization $\mathbb{R} \times Y^{ \pm}$. However, under evaluation at the boundary loops all these moduli spaces lead to degenerate chains in $\Sigma^{k}$, i.e. chains that factor through chains of lower dimension, and therefore do not appear in the master equation.

\section{Technical remarks}

Theorem 3.1 is conjectural at this point, as it requires analytic results even beyond those needed for the discussion of SFT. However, there are clear strategies for attacking the basic issues.

The discussion of coherent orientations should reduce to a careful combination of the results in [3] for the SFT case and [19] for the case of disks without punctures. There is one new phenomenon which has not been described in the literature yet, which deals with "self-gluing" at a transversal self-intersection of a boundary curve as in the operation $\Delta$. We expect to treat orientations completely in [9].

The necessary compactness results essentially reduce to a combination of standard SFT compactness [2] and the observation that our exactness assumption rules out bubbling 
of holomorphic spheres or disks. Then the remaining phenomena can be studied in terms of degenerations of domains, using a standard doubling trick. There is however one new feature that at the moment we do not know how to deal with properly: In dimension $n>3$ index considerations yield an upper bound on the number of boundary components on any curve with given set of asymptotics prescribed at the punctures and representing a fixed relative homology class. We do not expect such a result for $n=2,3$.

To achieve transversality for moduli spaces of punctured curves with boundaries, we hope to adapt the techniques in [12]. Note that the word transversality is used in two senses here, as it means both that moduli spaces are "transversally cut out" and that the resulting chains on string space have certain transversality properties needed to apply the string topology operations (cf. Section 4). The gluing results will require a combination of SFT-type gluing with boundary gluing as in [19].

\section{String topology}

In this section we discuss the new operations on the chains of string space introduced by Chas and Sullivan $[5,6]$.

Let $Q$ be a closed oriented smooth $n$-manifold, and define the free loop space $\Lambda Q$ as

$$
\Lambda:=\Lambda Q:=C^{0}\left(S^{1}, Q\right) .
$$

In the previous section, we also defined the closed string space $\Sigma Q$ of $Q$ by

$$
\Sigma:=\Sigma Q:=\Lambda Q / S^{1}
$$

For $k \geq 1$ let $\Sigma^{k}:=\Sigma \times \cdots \times \Sigma$ be the $k$-fold product.

A smooth $i$-chain in $\Sigma^{k}$ is a tuple $\left(K ; \sigma_{1}, \ldots, \sigma_{k}\right)$ where $K$ is some $i$-dimensional compact connected oriented manifold with corners, and for each $1 \leq i \leq k$ the $\sigma_{i}: P_{i} \rightarrow Q$ are smooth maps from the total space of some circle bundle $p_{i}: P_{i} \rightarrow$ $K$ into $Q$. To get a good set of chains, one actually needs to require that the domains, together with all the circle bundles, come with fixed embeddings into some $\mathbb{R}^{N}$. Denote by $\widetilde{C}_{i}\left(\Sigma^{k}\right)$ the $\mathbb{Q}$-vector space with basis the smooth $i$-chains in $\Sigma^{k}$, and by $C_{i}\left(\Sigma^{k}\right)$ the quotient space where we factor out the degenerate $i$-chains and identify $\left(-K, \sigma_{1}, \ldots, \sigma_{k}\right)$ with $-\left(K, \sigma_{1}, \ldots, \sigma_{k}\right)$, where $-K$ denotes $K$ with the opposite orientation.

Chas and Sullivan introduced partially defined coproduct and product operations

$$
\begin{gathered}
\delta: C_{i}(\Sigma) \rightarrow C_{i+2-n}(\Sigma \times \Sigma), \\
\mu: C_{i}(\Sigma \times \Sigma) \rightarrow C_{i+2-n}(\Sigma)
\end{gathered}
$$

as follows. To define the coproduct, let a smooth $i$-chain $(K ; \sigma) \in C_{i}(\Sigma)$ be given and consider the pullback bundle $p^{*}(P) \rightarrow P$. Note that this bundle has a tautological section, so that the induced map $p^{*} P \rightarrow Q$ can be viewed as a 
map $\widetilde{\sigma}: P \rightarrow \Lambda Q$. Denote by $K_{\delta \sigma} \subset P \times[0,1]$ the closure of the inverse image of the diagonal of $Q \times Q$ under the evaluation map

$$
\begin{aligned}
e v_{\sigma}: P \times(0,1) & \rightarrow Q \times Q \\
(p, t) & \mapsto(\widetilde{\sigma}(p)(0), \widetilde{\sigma}(p)(t)) .
\end{aligned}
$$

Assuming enough transversality, $K_{\delta \sigma}$ is a submanifold with corners of $P \times[0,1]$ of dimension $i+2-n$. Now the chain $\delta \sigma: K_{\delta \sigma} \rightarrow \Sigma \times \Sigma$ is defined by assigning to $(p, t) \in K_{\delta \sigma}$ the ordered pair of strings corresponding to the pair of loops $\left(\widetilde{\sigma}(p)_{\mid[0, t]}, \widetilde{\sigma}(p)_{\mid[t, 1]}\right)$. Note that the two corresponding circle bundles are trivial, since the point where we cut the old circle gives rise to a section.

Similarly, to define the product, we start from a smooth $i$-chain $\left(K ; \sigma_{1}, \sigma_{2}\right) \in$ $C_{i}\left(\Sigma^{2}\right)$ and consider the fiber product $\pi: P \rightarrow K$ of the two circle bundles. Then again the pull-backs $\pi^{*}\left(P_{i}\right) \rightarrow P$ have tautological sections, yielding two families of loops $\widetilde{\sigma}_{i}: P \rightarrow \Lambda Q, i=1,2$. Then we consider the evaluation map

$$
\begin{aligned}
e v: P & \rightarrow Q \times Q \\
p & \mapsto\left(\widetilde{\sigma}_{1}(p)(0), \widetilde{\sigma}_{2}(p)(0)\right) .
\end{aligned}
$$

Again assuming transversality, $K_{\mu \sigma}$ is a submanifold of $P$ of dimension $i+2-n$, and the chain $\mu \sigma: K_{\mu \sigma} \rightarrow \Sigma$ is defined by assigning to $p \in K_{\mu \sigma}$ the string corresponding to the concatenation of the two loops $\widetilde{\sigma}_{1}(p)$ and $\widetilde{\sigma}_{2}(p)$. The circle bundle involved is again trivial.

More generally, on $C_{*}\left(\Sigma^{k}\right)$ we can define operations $\delta_{r}$ and $\mu_{r_{1}, r_{2}}$ where the above operations are applied to the corresponding strings. Note that $\delta$ does not commute with the boundary operator, because of the possible "vanishing of small loops" (cf. Figure 4 ). However, after passing to the chains relative
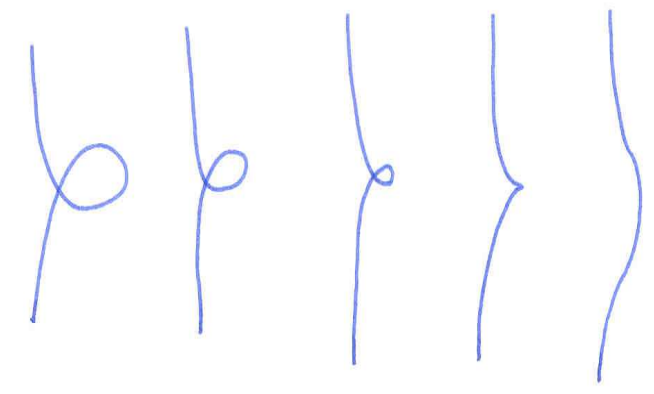

Figure 4: A small loop disappears

to the constant strings $C_{*}\left(\Sigma^{k}\right.$, const $\left._{k}\right)$ as in the previous section, we find that both operations behave well with respect to the boundary operator. Note that an application of $\mu$ to a pair of strings of which one is constant leads to a 
degenerate chain, so the operations indeed descend to the relative chains with our conventions.

Motivated by the grading conventions of SFT, we now consider the shifted chain complex

$$
\hat{\mathcal{C}}_{i, k}:=C_{i+k(3-n)}\left(\Sigma^{k}, \text { const }_{k}\right),
$$

and note that in this new grading the operations $\delta_{r}$ and $\mu_{r_{1}, r_{2}}$ have bidegrees $(-1,1)$ and $(-2(n-3)-1,-1)$, respectively. We let the symmetric group on $k$ letters act on $\hat{\mathcal{C}}_{i, k}$ by

$$
\rho \cdot\left(t \mapsto\left(\sigma_{1}(t), \ldots \sigma_{k}(t)\right)\right):=(\operatorname{sgn} \rho)^{3-n}\left(t \mapsto\left(\sigma_{\rho(1)}(t), \ldots \sigma_{\rho(k)}(t)\right)\right),
$$

and denote by $\mathcal{C}_{i, k}$ the quotient of $\hat{\mathcal{C}}_{i, k}$ by this action. Then the direct sum

$$
\mathcal{C}:=\mathbb{Q} \oplus \bigoplus_{k \geq 1, i \geq k(n-3)} \mathcal{C}_{i, k}
$$

forms an algebra with unit $\mathbf{1} \in \mathbb{Q}$ under the multiplication $\cdot$ defined as follows. Given chains $\sigma \in \mathcal{C}_{i, k}$ and $\tau \in \mathcal{C}_{j, l}$ we consider the new chain $\sigma \times \tau \in \mathcal{C}_{i+j, k+l}$ which is defined on the product of the domains and is given at the parameter value $(t, s)$ by the concatenation of the collections of strings $\sigma(t)$ and $\tau(s)$. Then we set

$$
\sigma \cdot \tau:=(-1)^{i l(3-n)} \sigma \times \tau .
$$

One can check that

$$
\sigma \cdot \tau=(-1)^{i j} \tau \cdot \sigma
$$

i.e. the multiplication is graded commutative. Denote by $\partial$ the usual boundary operator on $\mathcal{C}_{*, k}$, shifted by $(-1)^{k(3-n)}$. Then a short calculation shows that $\partial$ is a derivation of the algebra $\mathcal{C}$ with respect to the grading by the shifted dimension of chains. We now define an operation $\Delta: \mathcal{C} \rightarrow \mathcal{C}$ of bidegree $(-1,1)$ by $\Delta(\mathbf{1})=0$ and

$$
\Delta_{\mid \hat{\mathcal{C}}_{*, k}}:=\sum_{r=1}^{k}(-1)^{(r+k)(3-n)} \delta_{r}
$$

for $k \geq 1$, and an operation $\nabla: \mathcal{C} \rightarrow \mathcal{C}$ of bidegree $(-2(n-3)-1,-1)$ by $\nabla(\mathbf{1})=0$ and

$$
\nabla_{\mid \hat{\mathcal{C}}_{*, k}}:=\left\{\begin{array}{cc}
0 & \text { if } k=1 \\
\sum_{r_{1}<r_{2}}(-1)^{\left(r_{2}-1+k\right)(3-n)} \mu_{r_{1}, r_{2}} & \text { if } k>1
\end{array}\right.
$$

These are the operations appearing in the statement of Theorem 3.1. One checks that they are indeed well-defined on $\mathcal{C}$. The following proposition is implicit in [6] and will be proven in [10].

Proposition 4.1. On sufficiently transverse chains one has the following identities: 
(i) $\partial \Delta+\Delta \partial=0$

(ii) $\Delta^{2}=0$,

(iii) $\Delta\left(c_{1} \cdot c_{2}\right)=\Delta\left(c_{1}\right) \cdot c_{2}+(-1)^{\left|c_{1}\right|} c_{1} \cdot \Delta\left(c_{2}\right)$,

(iv) $\partial \nabla+\nabla \partial=0$,

(v) $\nabla^{2}=0$,

(vi)

$$
\begin{aligned}
& \nabla\left(c_{1} c_{2} c_{3}\right)= \\
& \quad \nabla\left(c_{1} c_{2}\right) c_{3}+(-1)^{\left|c_{1}\right|} c_{1} \nabla\left(c_{2} c_{3}\right)+(-1)^{\left|c_{2}\right|\left|c_{3}\right|} \nabla\left(c_{1} c_{3}\right) c_{2} \\
& -\nabla\left(c_{1}\right) c_{2} c_{3}-(-1)^{\left|c_{1}\right|} c_{1} \nabla\left(c_{2}\right) c_{3}-(-1)^{\left|c_{1}\right|+\left|c_{2}\right|} c_{1} c_{2} \nabla\left(c_{3}\right) .
\end{aligned}
$$

(vii) $\Delta \nabla+\nabla \Delta=0$

Denote by

$$
\mathcal{H}_{k}:=\mathcal{H}_{k}(\Sigma, Q):=H_{k+3-n}(\Sigma, Q)
$$

the shifted homology of string space relative to the constant strings. Part (i) and (iv) of the proposition imply that $\delta$ and $\mu$ give rise to operations

$$
\begin{aligned}
& \delta: \mathcal{H}_{*} \rightarrow \mathcal{H}_{*} \otimes \mathcal{H}_{*} \\
& \mu: \mathcal{H}_{*} \otimes \mathcal{H}_{*} \rightarrow \mathcal{H}_{*}
\end{aligned}
$$

of degrees -1 and $-2(n-3)-1$, respectively. The other properties listed in the proposition imply the following theorem of Chas and Sullivan [6].

Theorem 4.2 (Chas-Sullivan [6]). The operations $\mu$ and $\delta$ give the string homology $\mathcal{H}_{*}(\Sigma, Q)$ of any oriented manifold $Q$ the structure of an involutive Lie bialgebra of bidegree $(-1,-2(n-3)-1)$.

The precise definition of this concept will be given in the next section.

\section{Technical remarks}

The statement of Proposition 4.1 should be understood as follows: Each of the operations $\Delta, \nabla, \Delta^{2}, \nabla^{2}, \Delta \nabla$ and $\nabla \Delta$ has a domain of definition described in terms of certain jet transversality conditions for evaluation maps, and the stated identities hold on the intersections of the domains for the operations involved. This, as well as orientation issues, will be discussed in detail in [10].

Note that in order to give the statement of Theorem 3.1 rigorous meaning, one also needs to make sense of "diagonal terms" like $\nabla(\mathbf{L} \cdot \mathbf{L})$. These involve bracket-type operations for a chain with itself, which usually does not satisfy the transversality requirements. There are several solutions to this problem, see e.g. [18] for one which works in the context of holomorphic curves. 


\section{Some algebra}

In this section, we describe the abstract algebraic structures underlying both SFT and string topology. Throughout, we fix an integer $n$ and use the symbol $\hbar$ to denote a formal variable of degree $2(n-3)$. We also fix a field $\mathbb{K}$ of characteristic zero.

Definition. A (strictly commutative) $B V_{\infty}$-algebra is a pair $(A, D)$, where $A$ is a graded commutative algebra over $\mathbb{K}$ with unit and $D: A[[\hbar]] \rightarrow A[[\hbar]]$ is a linear map of degree -1 satisfying

(BV1) $D D=0$,

(BV2) $D$ admits an expansion

$$
D=\frac{1}{\hbar} \sum_{k=1}^{\infty} D^{k} \hbar^{k}
$$

where each $D^{k}: A \rightarrow A$ is a differential operator of order $\leq k$ on $A$, and

(BV3) $\mathrm{D}(1)=0$.

For the convenience of the reader, we recall the inductive definition of a differential operator over a morphism $m: A \rightarrow B$ of graded commutative algebras with unit: The zero map from $A$ to $B$ is a differential operator of order -1 , and a linear map $D: A \rightarrow B$ is a differential operator of order $\leq k$ over $m$ if for each $a \in A$ the map $x \mapsto D(x a)-D(x) m(a)$ is a differential operator of order $\leq k-1$. A differential operator on $A$ is by definition a differential operator over the identity $\mathbf{1}: A \rightarrow A$. For a polynomial algebra this reduces to the usual concept, where a differential operator of order $\leq k$ is one taking at most $k$ partial derivatives.

Since all the $\mathrm{BV}_{\infty}$-algebras appearing in these notes will be strictly commutative, we will permit ourselves to drop this qualifier from now on. Note that the requirement $D D=0$ can be written as a sequence of quadratic identities satisfied by the $D^{k}$ in the decomposition (12). The first few of these read

$$
D^{1} D^{1}=0, \quad D^{1} D^{2}+D^{2} D^{1}=0, \quad D^{1} D^{3}+D^{2} D^{2}+D^{3} D^{1}=0, \quad \text { etc. }
$$

In particular, $D^{1}$ is a derivation of $A$ and $D^{2}$ descends to the homology algebra $H\left(A, D^{1}\right)$ as a differential operator of order $\leq 2$ and square 0 , so that $\left(H\left(A, D^{1}\right), D^{2}\right)$ is a BV-algebra (cf. [20]). The deviation of $D^{2}$ from being a derivation is measured by the so-called BV-bracket

$$
[a, b]_{D}:=(-1)^{|a|}\left(D(a b)-D(a) b-(-1)^{|a|} a D(b)\right),
$$

which is an odd Poisson bracket on $H\left(A, D^{1}\right)$.

Next, we need the notion of a morphism of $\mathrm{BV}_{\infty}$-algebras. We will only give an ad hoc definition in the special case that the domain of the morphism is a 
free commutative algebra $A=S(V)$, where $S(V)$ denotes the symmetric tensor product of the graded vector space $V$. Given a linear map $\varphi: S(V) \rightarrow B[[\hbar]]$, we define a map $e^{\varphi}: S(V)[[\hbar]] \rightarrow B[[\hbar]]$ as

$$
\begin{aligned}
& e^{\varphi}\left(v_{1} \cdots v_{k}\right):= \\
& \quad \sum_{\ell=1}^{k} \frac{1}{\ell !} \sum_{i_{1}+\cdots+i_{\ell}=k} \frac{1}{i_{1} ! \cdots i_{\ell} !} \sum_{\rho \in S_{k}} \varepsilon(\rho) \varphi\left(v_{\rho(1)} \cdots v_{\rho\left(i_{1}\right)}\right) \cdots \varphi\left(v_{\rho\left(k-i_{\ell}+1\right)} \cdots v_{\rho(k)}\right),
\end{aligned}
$$

where $\varepsilon(\rho)$ is the sign one gets from rearranging the $v_{j}$ according to the permutation $\rho$, and the map is extended to $S(V)[[\hbar]]$ by $\mathbb{K}[[\hbar]]$-linearity.

Definition. A $B V_{\infty}$-morphism from a free $\mathrm{BV}_{\infty}$-algebra $\left(A=S(V), D_{A}\right)$ to a $\mathrm{BV}_{\infty}$-algebra $\left(B, D_{B}\right)$ is a linear map $\varphi: A \rightarrow B[[\hbar]]$ of degree 0 satisfying

$($ BVmor1) $\varphi(1)=0$,

(BVmor2) $e^{\varphi} D_{A}=D_{B} e^{\varphi}$, and

(BVmor3) $\varphi$ admits an expansion

$$
\varphi=\frac{1}{\hbar} \sum_{k=1}^{\infty} \varphi^{k} \hbar^{k}
$$

where each $\varphi^{k}: A \rightarrow B$ is a differential operator of order $\leq k$ over the zero morphism, i.e. $\varphi^{k}\left(v_{1} \cdots v_{l}\right)=0$ for $l>k$.

An augmentation of a free $\mathrm{BV}_{\infty}$-algebra is a morphism from $\left(A=S(V), D_{A}\right)$ to the trivial $\mathrm{BV}_{\infty}$-algebra $(\mathbb{K}, 0)$.

As before for $D$, the equation in (BVmor2) can be expanded in powers of $\hbar$. The leading order term will have the form

$$
e^{\varphi^{1}} D_{A}^{1}=D_{B}^{1} e^{\varphi^{1}} .
$$

Note that since $\varphi^{1}$ can only be non-zero on $V \cong S^{1}(V) \subset S(V)$, the associated map $e^{\varphi^{1}}: S(V) \rightarrow B$ is an algebra morphism. The above equation then says that it is in fact a morphism of differential graded algebras from $\left(A, D_{A}^{1}\right)$ to $\left(B, D_{B}^{1}\right)$, and so it induces a morphism between the corresponding homology algebras. We also remark that if $\beta: A[[\hbar]] \rightarrow \mathbb{K}[[\hbar]]$ is an augmentation of the $\mathrm{BV}_{\infty}$-algebra $\left(A, D_{A}\right)$, then $e^{\beta^{1}}$ is an augmentation of the differential graded algebra $\left(A, D_{A}^{1}\right)$.

Now let $(A=S(V), D)$ be a free $\mathrm{BV}_{\infty}$-algebra and suppose we are given an augmentation $\beta: S(V) \rightarrow \mathbb{K}[[\hbar]]$. From it we construct a linear map $\Phi=\Phi^{\beta}$ : $A \rightarrow A$ as

$$
\Phi\left(v_{1} \cdots v_{k}\right)=v_{1} \cdots v_{k}+\sum_{l=1}^{k} \frac{1}{l !(k-l) !} \sum_{\rho \in S_{k}} \varepsilon(\rho) e^{\beta}\left(v_{\rho(1)} \cdots v_{\rho(l)}\right) v_{\rho(l+1)} \cdots v_{\rho(k)} .
$$


One can prove by induction that there is a unique map $f: S(V) \rightarrow S(V)[[\hbar]]$ such that $\Phi=e^{f}$, and $f$ satisfies properties (BVmor1) and (BVmor3) of a $\mathrm{BV}_{\infty^{-}}$ morphism. One also proves that $\Phi$ is invertible. Moreover, by construction we have $\pi_{0} \Phi=e^{\beta}$, where $\pi_{0}: S(V) \rightarrow V^{0} \cong \mathbb{K}$ is the projection to the constants. Now one can introduce a twisted $B V_{\infty}$-operator $D^{\beta}$ on $A$ by setting

$$
D^{\beta}:=\Phi D \Phi^{-1} .
$$

This new $\mathrm{BV}_{\infty}$-operator on $A$ has the additional property that it has no constant terms, in the sense that $\pi_{0} D^{\beta}(a)=0$ for any $a \in A[[\hbar]]$. This is because

$$
\pi_{0} D^{\beta}(a)=\pi_{0} \Phi D \Phi^{-1}(a)=e^{\beta} D\left(\Phi^{-1}(a)\right)=0
$$

since $\beta$ was an augmentation. It is now clear by construction that $\Phi$ is an isomorphism between the $\mathrm{BV}_{\infty}$-algebras $(A, D)$ and $\left(A, D^{\beta}\right)$.

$\mathrm{BV}_{\infty}$-operators without constant terms on $S(V)$ are really nice objects, as they can be linearized to give an "infinity involutive Lie bialgebra structure" on $V$. We will not do this here, but in the remainder of this section we explain in detail the construction of the linearized homology and outline the construction of the Lie bialgebra structure it carries.

Expanding $\Phi=\sum_{k=0}^{\infty} \Phi^{k} \hbar^{k}$ in powers of $\hbar$, the leading order term is a map $\Phi^{0}: A \rightarrow A$ which has the form

$$
\begin{aligned}
& \Phi^{0}\left(v_{1} \cdots v_{k}\right) \\
& \quad=v_{1} \cdots v_{k}+\sum_{l=1}^{k} \frac{1}{l !(k-l) !} \sum_{\rho \in S_{k}} \varepsilon(\rho) e^{\beta^{1}}\left(v_{\rho(1)} \cdots v_{\rho(l)}\right) v_{\rho(l+1)} \cdots v_{\rho(k)} \\
& \quad=v_{1} \cdots v_{k}+\sum_{l=1}^{k} \frac{1}{l !(k-l) !} \sum_{\rho \in S_{k}} \varepsilon(\rho) \beta^{1}\left(v_{\rho(1)}\right) \cdots \beta^{1}\left(v_{\rho(l)}\right) v_{\rho(l+1)} \cdots v_{\rho(k)} \\
& =\left(v_{1}+\beta^{1}\left(v_{1}\right)\right) \cdots\left(v_{k}+\beta^{1}\left(v_{k}\right)\right),
\end{aligned}
$$

so it is an algebra automomorphism. As above for $\Phi$, one can check that the constant term $\pi_{0} \Phi^{0}$ equals $e^{\beta^{1}}: A \rightarrow \mathbb{K}$, which is an augmentation of the differential graded algebra $\left(A, D^{1}\right)$. It is now standard [7] to consider the twisted derivation

$$
\partial^{\beta}:=\Phi^{0} D^{1}\left(\Phi^{0}\right)^{-1},
$$

which obviously still squares to 0 . One can check that in fact $\partial^{\beta}$ is nothing but the leading term $\left(D^{\beta}\right)^{1}$ in the expansion of $D^{\beta}$. Being a derivation, it is completely determined by the restriction $\left.\partial^{\beta}\right|_{V \subset S(V)}$, which can be expanded as

$$
\left.\partial^{\beta}\right|_{V}=\partial_{0}^{\beta}+\partial_{1}^{\beta}+\partial_{2}^{\beta}+\ldots
$$

with $\partial_{k}^{\beta}: V \rightarrow S^{k}(V)$. Now note that $\partial_{0}^{\beta}=e^{\beta^{1}} D^{1}\left(\Phi_{0}\right)^{-1}=0$. It follows that $\partial^{\text {lin }}:=\partial_{1}^{\beta}: V \rightarrow V$ defines a differential. We call its homology the 
linearized homology of the augmented $B V_{\infty}$-algebra $(A, D, \beta)$ and denote it by $H^{\operatorname{lin}}(A, D, \beta)$.

One can view the higher order terms $\partial_{k}^{\beta}: V \rightarrow S^{k}(V)$ in the expansion as operations which (after introducing suitable signs) combine into an odd co- $\mathrm{L}_{\infty^{-}}$ structure on $V$. In particular, $\partial_{2}^{\beta}: V \rightarrow S^{2}(V)$ gives rise to a map

$$
\delta: V \rightarrow V \otimes V, \quad v \mapsto(\iota \otimes \mathbf{1}) c\left(\partial_{2}^{\beta}(v)\right),
$$

where $\iota(w)=(-1)^{|w|} w$ and $c: S^{2} V \rightarrow V \otimes V$ is given on generators as $c\left(v_{1} v_{2}\right)=$ $v_{1} \otimes v_{2}+(-1)^{\left|v_{1}\right|\left|v_{2}\right|} v_{2} \otimes v_{1}$. One can check that $\delta$ descends to the linearized homology and gives it the structure of a co-Lie algebra of degree -1 , in the sense of the following

Definition. A co-Lie algebra of degree $d$ is a graded vector space $V$ with a linear map $\delta: V \rightarrow V \otimes V$ of degree $d$ such that

(i)

$$
\tau_{d} \delta=-\delta,
$$

where $\tau_{d}: V^{\otimes 2} \rightarrow V^{\otimes 2}$ is given on decomposable elements as

$$
\tau_{d}\left(v_{1} \otimes v_{2}\right):=(-1)^{\left(\left|v_{1}\right|+d\right)\left(\left|v_{2}\right|+d\right)} v_{2} \otimes v_{1},
$$

and

(ii)

$$
0=\left(1+\rho_{d}+\rho_{d}^{2}\right)(\delta \otimes \mathbf{1}) \delta: V \rightarrow V^{\otimes 3},
$$

where $\rho_{d}: V^{\otimes 3} \rightarrow V^{\otimes 3}$ is given on decomposable elements as

$$
\rho_{d}\left(v_{1} \otimes v_{2} \otimes v_{3}\right)=(-1)^{\left(\left|v_{1}\right|+\left|v_{2}\right|\right)\left(\left|v_{3}\right|+d\right)} v_{3} \otimes v_{1} \otimes v_{2} .
$$

To get a Lie bracket on the linearized homology, we expand the twisted $\mathrm{BV}_{\infty^{-}}$ operator $D^{\beta}$ as

$$
D^{\beta}=\frac{1}{\hbar} \sum_{k} D^{\beta, k} \hbar^{k}
$$

and further consider the component $D_{1}^{\beta, 2}: S^{2}(V) \rightarrow V$ of $D^{\beta, 2}$. Then one can check that the map

$$
\mu: V \otimes V \rightarrow V, \quad \mu\left(v_{1}, v_{2}\right)=(-1)^{\left|v_{1}\right|} D_{1}^{\beta, 2}\left(v_{1} v_{2}\right)
$$

descends to the linearized homology and gives it the structure of a graded Lie algebra of degree $-2(n-3)-1$, in the following sense.

Definition. A Lie algebra of degree $d$ is a graded vector space $V$ together with a linear map $\mu: V \otimes V \rightarrow V$ of degree $d$ such that

(i) $\mu \tau_{d}=-\mu$, and 
(ii) $0=\mu(\mu \otimes \mathbf{1})\left(1+\rho_{d}+\rho_{d}^{2}\right): V^{\otimes 3} \rightarrow V$.

Here $\tau_{d}$ and $\rho_{d}$ are as in the previous definition.

In fact, our situation is even better, since the compatibility conditions in the $\mathrm{BV}_{\infty}$-algebra force a strong link between the Lie algebra and co-Lie algebra structures on linearized homology.

Definition. A Lie bialgebra of bidegree $\left(d_{1}, d_{2}\right)$ is a graded vector space $V$ with linear maps $\delta: V \rightarrow V \otimes V$ of degree $d_{1}$ and $\mu: V \otimes V \rightarrow V$ of degree $d_{2}$ such that

(i) $(V, \delta)$ is a co-Lie algebra of degree $d_{1}$,

(ii) $(V, \mu)$ is a Lie algebra of degree $d_{2}$, and

(iii) $\delta$ and $\mu$ satisfy Drinfeld compatibility, i.e.

$$
\begin{aligned}
\delta \mu(a, b)= & \sum a_{1} \otimes \mu\left(a_{2}, b\right)+\mu\left(a, b_{1}\right) \otimes b_{2} \\
& +\sum(-1)^{|a||b|+|a|+|b|}\left(\mu\left(b, a_{1}\right) \otimes a_{2}+b_{1} \otimes \mu\left(b_{2}, a\right)\right),
\end{aligned}
$$

where $\delta(a)=\sum a_{1} \otimes a_{2}$ and $\delta(b)=\sum b_{1} \otimes b_{2}$.

The Lie bialgebra is called involutive if moreover $0=\mu \delta: V \rightarrow V$.

Note that if $\delta$ and $\mu$ have different parity, then involutivity is automatically satisfied on the part of the Lie bialgebra with grading $\equiv d_{1} \bmod 2$.

Our discussion on augmentations can now be summarized in the following claim.

Proposition 5.1. Any augmentation $\beta$ of a free $B V_{\infty}$-algebra $(A=S(V), D)$ gives rise to a linearized homology $H^{\operatorname{lin}}(A, D, \beta)$ which has the structure of an involutive Lie bialgebra of bidegree $(-1,-2(n-3)-1)$.

A morphism between augmented $\mathrm{BV}_{\infty}$-algebras $\left(A, D_{A}, \alpha\right)$ and $\left(B, D_{B}, \beta\right)$ is a $\mathrm{BV}_{\infty}$-morphism $\varphi$ which in addition satisfies

$$
e^{\alpha}=e^{\beta} \circ e^{\varphi} .
$$

The following statement will be proven in [8].

Proposition 5.2. Any morphism between augmented $B V_{\infty}$-algebras $\left(A, D_{A}, \alpha\right)$ and $\left(B, D_{B}, \beta\right)$ gives rise to a chain map for the linearized complexes which induces a morphism of involutive Lie bialgebras in homology.

The following concepts will become important in Section 8 . 
Definition. A Maurer-Cartan element in a $\mathrm{BV}_{\infty}$-algebra $(A, D)$ is an element $a \in \frac{1}{\hbar} A[[\hbar]]$ of degree zero satisfying

$$
D\left(e^{a}\right)=0 .
$$

Given a Maurer-Cartan element $a \in \frac{1}{\hbar} A[[\hbar]]$, one defines the twisted $B V_{\infty}$ operator

$$
D_{a}: A[[\hbar]] \rightarrow A[[\hbar]], \quad b \mapsto e^{-a} D\left(e^{a} b\right) .
$$

One can check that $D_{a}$ indeed maps between the spaces given and $\left(A, D_{a}\right)$ is again a $\mathrm{BV}_{\infty}$-algebra. In the special case that the original $\mathrm{BV}$-operator $D$ had the property that $D^{k}=0$ for all $k \geq 3$, the condition $D\left(e^{a}\right)=0$ simplifies to

$$
D a+\frac{\hbar}{2}[a, a]_{D}=0
$$

\section{Technical remarks}

The proper concept of $\mathrm{BV}_{\infty}$-algebra is more general than the one presented here; it is discussed by Tamarkin and Tsygan [26]. What we consider here is essentially equivalent to what Kravchenko [23] calls homotopy BV-algebras.

In the augmented free case it is more appropriate to discuss everything in terms of involutive Lie bialgebras up to homotopy, since in this case our $\mathrm{BV}_{\infty}$-algebras are just cobar constructions on the co-Lie part of the structure. The relevant algebra will be developed in [8].

However, the object that arises naturally from the SFT of a contact manifold without filling is only a weak version of such involutive $\mathrm{BL}_{\infty}$ algebras, meaning that in general one does have $n$-to-0 operations. We do not know how to properly treat them on the linear level, i.e. without going to the cobar construction.

One essential aspect which is completely missing here is the discussion of homotopies. These are necessary to properly formulate the invarince properties of the various algebraic objects constructed in both SFT and string topology, see [8].

\section{Back to geometry}

In this section, we take another look at SFT and string topology and describe them using the language developed in the previous section.

We have seen in Section 2 that to a closed contact manifold $\left(Y^{2 n-1}, \lambda\right)$ and a suitable choice of almost complex structure on $\mathbb{R} \times Y$ one can associate an element $\mathbf{H} \in \frac{1}{\hbar} \mathcal{W}$, where $\mathcal{W}$ is the graded Weyl algebra constructed from the (good) closed Reeb orbits of $\lambda$. In particular, $\mathcal{W}$ contains the graded commutative subalgebra $\mathcal{A}$ of polynomials in the variables $q_{\gamma}$.

We claim that the differential operator $\mathbf{D}=\mathbf{D}_{\mathrm{SFT}}:=\overrightarrow{\mathbf{H}}: \mathcal{A}[[\hbar]] \rightarrow \mathcal{A}[[\hbar]]$ 
defined as in Section 2 via the replacements

$$
p_{\gamma} \mapsto \kappa_{\gamma} \hbar \frac{\vec{\partial}}{\partial q_{\gamma}}
$$

gives $\mathcal{A}$ the structure of a $\mathrm{BV}_{\infty}$-algebra as defined in the previous section. Indeed, the star product of the Weyl algebra exactly models the behavior of our differential operators under composition, so that the condition $\mathbf{D D}=0$ is a direct translation of $\mathbf{H} * \mathbf{H}=0$. Next note that each differentiation contributes one power of $\hbar$, so that the coefficient of $\hbar^{k-1}$ in the expansion of $\mathbf{D}$ is a differential operator of order $\leq k$. Finally the condition $\mathbf{D}(1)=0$ follows from the fact that there are no curves without positive punctures in $\mathbb{R} \times Y$, so every term of $\mathbf{H}$ contains at least one $p$ and $\mathbf{D}$ does not have any zero order terms as a differential operator.

Tracing through the definitions, one finds that the holomorphic curves which correspond to the term $\mathbf{D}^{k}$ in the expansion of $\mathbf{D}$ are precisely those for which the number of positive punctures and the genus add up to $k$. In particular, we find that $\mathbf{D}^{1}$ corresponds to curves of genus zero with exactly one positive puncture, so that $H_{*}\left(\mathcal{A}, \mathbf{D}_{\mathrm{SFT}}^{1}\right)$ is just the usual contact homology of $Y$. The $\mathrm{BV}$-operator $\mathbf{D}^{2}$ consists of two terms: a differential operator of order two corresponding to genus zero curves with two positive punctures and a derivation corresponding to curves of genus one with one positive puncture. Note that, in particular, the corresponding BV-bracket is determined just by the genus zero curves.

Next we discuss the morphism arising from an exact symplectic cobordism $(X, \omega)$ from $\left(Y^{+}, \lambda^{+}\right)$to $\left(Y^{-}, \lambda^{-}\right)$. Indeed, the potential $\mathbf{F}$ of the cobordism $X$ is a power series in the variables $p_{\gamma}^{+}$and $\hbar$ with coefficients polynomial in the $q_{\gamma}^{-}$. Analogously to the above discussion for $\mathbf{H}$, it gives rise to a map $\overrightarrow{\mathbf{F}}: \mathcal{A}^{+} \rightarrow \mathcal{A}^{-}[[\hbar]]$. We claim that this map is a $\mathrm{BV}_{\infty}$-morphism as defined in the previous section.

Again each term of $\mathbf{F}$ contains at least one $p^{+}$, so that we have $\overrightarrow{\mathbf{F}}(1)=0$. Simple algebraic manipulations show that the master equation of Theorem 2.2 is equivalent to the fact that

$$
e^{\overrightarrow{\mathbf{F}}} \mathbf{D}^{+}=\mathbf{D}^{-} e^{\overrightarrow{\mathbf{F}}}
$$

The statement about the order of the differential operators in the expansion of $\overrightarrow{\mathbf{F}}$ with respect to $\hbar$ follows as in the case of $\mathbf{D}$. Also, as for $\mathbf{D}$, the curves contributing to $\mathbf{F}^{k}$ are precisely those for which the number of positive punctures and the genus add up to $k$. In particular, the chain map yielding a morphism between the contact homologies is given by curves of genus zero with exactly one positive puncture.

Augmentations arise in a geometric way by considering symplectic cobordisms with only a positive end, since the $\mathrm{BV}_{\infty}$-algebra associated to the empty contact manifold is $(\mathbb{K}, 0)$. From Proposition 5.1 we then obtain 
Theorem 6.1. Every exact symplectic filling $(X, d \lambda)$ of a closed contact manifold $\left(Y^{2 n-1}, \lambda\right)$ gives rise to a linearized contact homology $H C^{\operatorname{lin}}(X, \lambda)$ which has the structure of an involutive Lie bialgebra of bidegree $(-1,-2(n-3)-1)$.

To get a feeling for this invariant of the symplectic filling, it may be instructive to describe the operations involved in the definition of linearized contact homology in terms of the broken holomorphic curves they count.

We start by looking at the boundary operator $\partial^{\text {lin }}$ of linearized contact homology. We claim that it counts configurations of curves as depicted in Figure 5. Here the numbers written in the various non-trivial components denote the in-

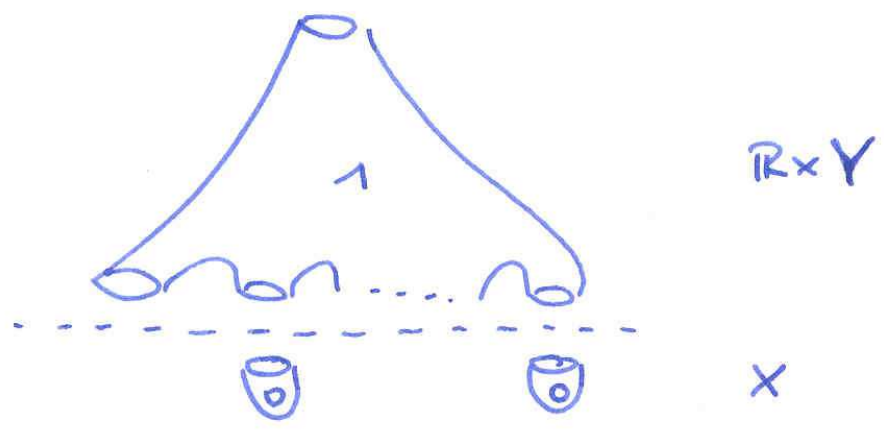

Figure 5: The linearized boundary operator

dex of the corresponding family of curves. To understand this picture, note first that the augmentation is given by the potential $\mathbf{F}$ of the filling. In particular, the augmentation $e^{\overrightarrow{\mathbf{F}^{1}}}:\left(\mathcal{A}, \mathbf{D}_{\mathrm{SFT}}^{1}\right) \rightarrow \mathbb{Q}$ of the underlying differential graded algebra is determined by the count of finite energy planes in $X$ of index 0 . The algebra automorphism $\Phi^{0}: \mathcal{A} \rightarrow \mathcal{A}$ is given on generators as $\Phi^{0}\left(q_{\gamma}\right)=q_{\gamma}+\overrightarrow{\mathbf{F}^{1}}\left(q_{\gamma}\right)$. Recall that $\partial^{\text {lin }}=\Phi^{0} \mathbf{D}^{1}\left(\Phi^{0}\right)^{-1}$, so that

$$
\partial^{\overrightarrow{\mathbf{F}}}\left(q_{\gamma}\right)=\Phi^{0} \mathbf{D}^{1}\left(q_{\gamma}-\overrightarrow{\mathbf{F}}^{1}\left(q_{\gamma}\right)\right)=\Phi^{0} \mathbf{D}^{1}\left(q_{\gamma}\right) .
$$

Now one can see that the linear part $\partial^{\text {lin }}$ corresponds precisely to configurations as in Figure 5. We remark in passing that the constant term in $\partial^{\overrightarrow{\mathbf{F}}}\left(q_{\gamma}\right)$ counts exactly the rigid configurations of the type pictured in Figure 6.

The fact that it is zero corresponds to the geometric fact that these configurations together form the oriented boundary of the corresponding moduli space of finite energy planes in $X$ of index 1 asymptotic to $\gamma$.

The same reasoning as for the linearized boundary operator $\partial^{\text {lin }}$ shows that the coefficients of the cobracket on linearized homology are given by the counts of configurations as depicted in Figure 7. 


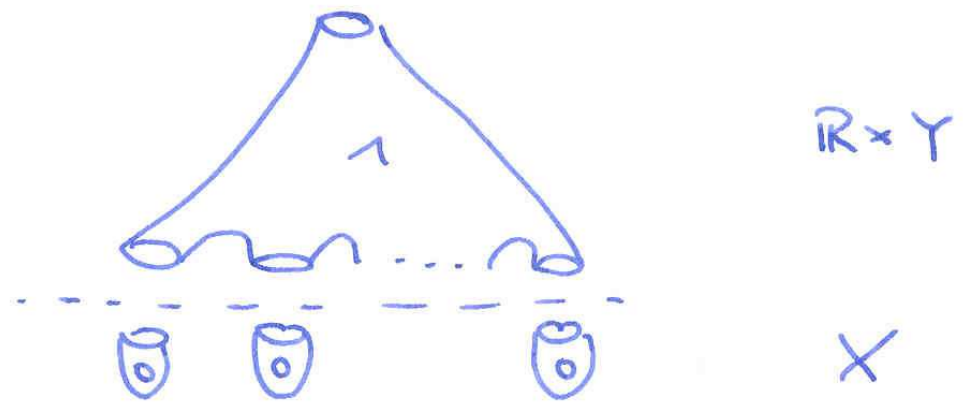

Figure 6: The constant term
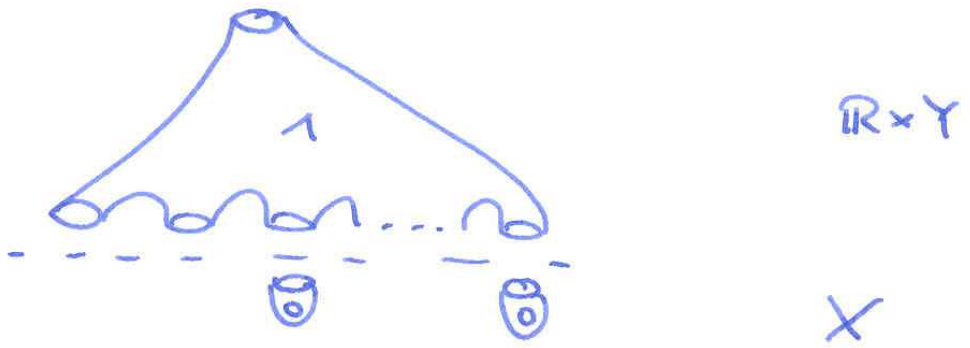

Figure 7: The cobracket on linearized homology

Something more interesting happens for the bracket, where we get the two types of pictures seen in Figure 8.

We next turn to string topology. Here things are somewhat more complicated, as the operations $\Delta$ and $\nabla$ are only partially defined. Still the compatibility conditions listed in Proposition 4.1 suggest that $\mathbf{D}_{\text {string }}:=\partial+\Delta+\hbar \nabla: \mathcal{C}[[\hbar]] \rightarrow$ $\mathcal{C}[[\hbar]]$ is reasonably viewed as a (partially defined) $\mathrm{BV}_{\infty}$-operator on $\mathcal{C}$. Note that in our grading conventions it is indeed homogeneous of degree -1 .

Now assume $(X, d \lambda)$ is an exact filling of the contact manifold $(Y, \lambda)$ and $Q \subset X$ is an exact Lagrangian. In this situation the master equation of Theorem 3.1 simplifies to

$$
\mathbf{D}_{\text {string }} e^{\mathbf{L}}=e^{\mathbf{L}} \overleftarrow{\mathbf{H}^{+}}
$$

So replacing $\mathbf{F}$ by $\mathbf{L}$ in the discussion before, we find that $\overrightarrow{\mathbf{L}}: \mathcal{A} \rightarrow \mathcal{C}[[\hbar]]$ defines a $\mathrm{BV}_{\infty}$-morphism between the $\mathrm{BV}_{\infty}$-algebra $\left(\mathcal{A}, \mathbf{D}_{\mathrm{SFT}}\right)$ of the contact manifold 

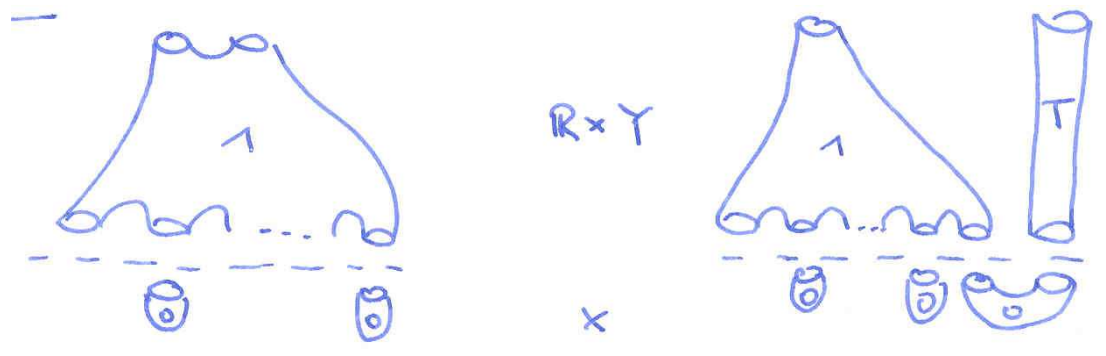

Figure 8: The bracket on linearized homology

$(Y, \lambda)$ and the (partial) $\mathrm{BV}_{\infty}$-algebra $\left(\mathcal{C}, \mathbf{D}_{\text {string }}\right)$ constructed from the string topology of $Q$.

One can actually do better. Split $\mathbf{L}=\mathbf{F}+\mathbf{L}^{\prime}$, where $\mathbf{F}$ is the potential of the filling $(X, d \lambda)$ of $Y$, i.e. the part of $\mathbf{L}$ corresponding to curves with no boundary. It gives an augmentation $\overrightarrow{\mathbf{F}}$ for $\left(\mathcal{A}, \mathbf{D}_{\mathrm{SFT}}\right)$, leading to the twisted $\mathrm{BV}_{\infty}$-operator

$$
\mathbf{D}_{\mathrm{SFT}}^{\mathbf{F}}:=\overrightarrow{e^{\mathbf{F}}} \mathbf{D}_{\mathrm{SFT}} e^{-\overrightarrow{\mathbf{F}}}
$$

on $\mathcal{A}$. As discussed before, this twisted $\mathrm{BV}_{\infty}$-operator does not have constant terms, so it admits a linearization. Moreover, denoting by $\mathcal{A}^{1} \subset \mathcal{A}$ the linear polynomials in the $q$ 's, i.e. the vector space generated by the good closed Reeb orbits, we obtain

Theorem 6.2. The part $\mathbf{L}_{1}^{1}$ of $\mathbf{L}^{\prime}$ corresponding to curves with one puncture and one boundary component gives rise to a chain map from $\mathcal{A}^{1}$ to $\mathcal{C}^{1}=C_{*}(\Sigma Q, Q)$ which induces a morphism of involutive graded Lie bialgebras

$$
H C^{\operatorname{lin}}(X, \lambda) \rightarrow H(\Sigma Q, Q) .
$$

There should be a more conceptual form of Theorem 6.2 giving a morphism of the chain-level structures.

\section{Technical remarks}

The proof of Theorem 6.1 uses only the algebraic properties of SFT described in [16]. While Theorem 6.2 is a similar consequence of Theorem 3.1, in [9] we will provide a complete, more direct proof which is independent of the master equation.

\section{Cotangent bundles}

Here we discuss the important special case of cotangent bundles. More precisely, let $X=T^{*} Q$ with its canonical symplectic form and $Q \subset X$ be the zero 
section. In this context, Theorem 6.2 yields a morphism of involutive graded Lie bialgebras

$$
\psi: H C^{\operatorname{lin}}\left(T^{*} Q, \lambda\right) \rightarrow H(\Sigma Q, Q) .
$$

The main result of this section is

Theorem 7.1. The map $\psi: H C^{\operatorname{lin}}\left(T^{*} Q, \lambda\right) \rightarrow H(\Sigma Q, Q)$ is an isomorphism of involutive graded Lie bialgebras.

The proof of this theorem is based on an action/length estimate for holomorphic curves. For this, we pick a Riemannian metric on $Q$ and let $Y=S^{*} Q$ be the unit cotangent bundle for this metric with the canonical 1-form $\lambda=p d q$. Then closed Reeb orbits $\gamma$ on $(Y, \lambda)$ are lifts of closed geodesics on $Q$ (again denoted by $\gamma$ ) and their action $\int_{\gamma} \lambda$ equals the length length $(\gamma)$ of the geodesic.

We pick a compatible almost complex structure $J_{\rho}$ on $T^{*} Q$ of the form

$$
J_{\rho}: \frac{\partial}{\partial q_{i}} \mapsto-\rho(|p|) \frac{\partial}{\partial p_{i}}, \quad \rho(|p|) \frac{\partial}{\partial p_{i}} \mapsto \frac{\partial}{\partial q_{i}},
$$

where $q_{i}$ are geodesic normal coordinates on $Q$ and $p_{i}$ are dual coordinates. Here $\rho:[0, \infty) \rightarrow(0, \infty)$ is a non-decreasing function with $\rho(r) \equiv 1$ near $r=0$ and $\rho(r)=r$ for $r$ large. For this almost complex structure, every $T$-periodic geodesic $\gamma$ gives rise to two natural $J_{\rho}$-holomorphic curves: the cylinder over $\gamma$

$$
\mathbb{R} \times \mathbb{R} / \mathbb{Z} \rightarrow T^{*} Q, \quad(s, t) \mapsto(\gamma(T t), g(s) \dot{\gamma}(T t)),
$$

where $g: \mathbb{R} \rightarrow \mathbb{R}$ is the solution of $g^{\prime}(s)=T \rho(s)$ with $g(0)=0$, and its restriction to $[0, \infty) \times \mathbb{R} / \mathbb{Z}$ which we call the half-cylinder over $\gamma$.

Lemma 7.2. Let $J_{\rho}$ be as above. Then for any $J_{\rho}$-holomorphic curve $f$ in $T^{*} Q$ with finitely many positive punctures asymptotic to closed geodesics $\gamma_{1}, \ldots, \gamma_{s}$ and with finitely many boundary loops $\sigma_{1}, \ldots, \sigma_{k}$ on $Q$ we have

$$
\sum_{i=1}^{k} \operatorname{length}\left(\sigma_{i}\right) \leq \sum_{j=1}^{s} \operatorname{length}\left(\gamma_{j}\right) .
$$

Equality holds if and only if $f$ is a branched cover of the half-cylinder over a closed geodesic.

The idea of the proof is the following. Consider the 1-form

$$
\lambda:=\frac{p d q}{|p|}
$$

on $T^{*} Q \backslash Q$. A straightforward computation shows that $f^{*} d \lambda \geq 0$ for every $J_{\rho}$-holomorphic curve $f$. Moreover, the integral of $f^{*} \lambda$ over a small loop around the $j$-th positive puncture converges to length $\left(\gamma_{i}\right)$ as the loop shrinks to a point (this is immediate), and the integral of $f^{*} \lambda$ over a loop around the $i$-th boundary 
component converges to length $\left(\sigma_{i}\right)$ as the loop converges to the boundary (this is a bit tricky). Now the inequality follows from Stokes' theorem:

$$
0 \leq \int_{f} d \lambda=\sum_{j=1}^{s} \operatorname{length}\left(\gamma_{j}\right)-\sum_{i=1}^{k} \operatorname{length}\left(\sigma_{i}\right)
$$

Moreover, this argument shows that equality holds iff $f^{*} d \lambda \equiv 0$, which happens precisely for branched covers of orbit half-cylinders. To make this idea rigorous, however, some care has to be taken when $f$ hits $Q$ at interior points since $f^{*} d \lambda$ is not defined there.

Given Lemma 7.2, the proof of Theorem 7.1 follows a standard argument, using the filtration by actions on the SFT side and by length on the string topology side and applying the Five Lemma .

There is, however, one important point. In the chain complex for linearized contact homology, only good Reeb orbits appear. One checks that in the present context a Reeb orbit is bad if and only if the stable bundle of the corresponding $S^{1}$-family of geodesics in $Q$, viewed as a critical manifold of the energy functional, is non-orientable. In this case, the local Morse homology vanishes [24], so that it does not contribute to the homology on the string topology side either.

\section{An Example: Surfaces of genus $>2$}

To illustrate Theorem 7.1, we discuss the special case where $Q$ is a closed oriented surface of genus $g \geq 2$. In this case the space of strings in each non-trivial free homotopy class is contractible, so that with our grading conventions the string homology is concentrated in degree -1 and equals

$$
\mathcal{H}_{*}(\Sigma, Q)=\mathcal{H}_{-1}(\Sigma Q, Q)=\bigoplus_{0 \neq \gamma \in\left[S^{1}, Q\right]} \mathbb{Q}\langle\gamma\rangle
$$

The bracket and cobracket on $\mathcal{H}_{*}$ in this case were discovered by Goldman [21] and Turaev [27], respectively, and were an important precursor for the definition of string topology by Chas and Sullivan. Note that here the abstract definitions given in Section 4 reduce to the following simple prescription: For the bracket, represent the two given free homotopy classes by curves in general position, so that they intersect transversally in finitely many points. Now each intersection point contributes one summand to the bracket. It is simply the free homotopy class obtained by concatenation of the two loops based there, with a sign obtained by comparing the ordered set of tangent vectors with the orientation of the surface, see Figure 9.

Similarly, for the cobracket, represent a given free homotopy class by a curve in general position with itself. Then the cobracket has two summands for each self-intersection point. They are given by the two ordered pairs of the classes obtained by splitting the closed curve into two, again each with the sign obtained by comparing the ordered set of tangent vectors to the surface orientation, see 

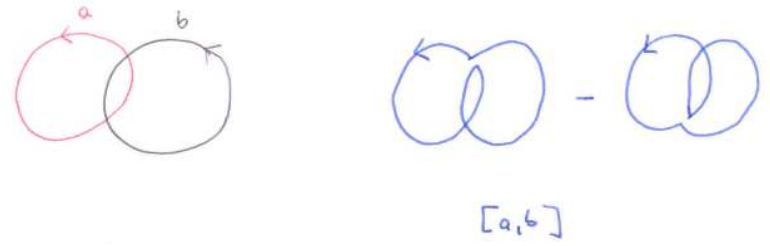

Figure 9: The bracket for curves on a surface

Figure 10. Here the color coding is such that the red string is the first and the black string is the second. Note, in particular, that the bracket and cobracket can be effectively computed for any given input.
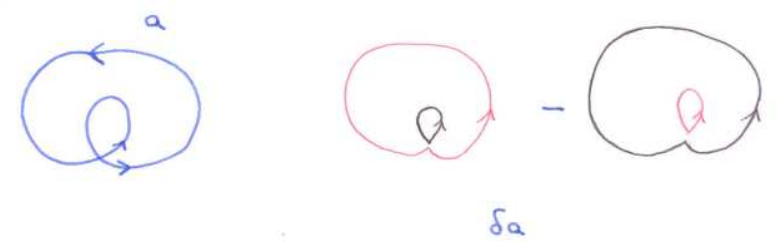

Figure 10: The cobracket for curves on a surface

Our next goal is to translate this topological information into existence results for holomorphic curves in $\mathbb{R} \times S^{*} Q$. For this we choose some Riemannian metric of negative curvature on our surface $Q$. Then all closed Reeb orbits are noncontractible and their Morse indices, and hence their Conley-Zehnder indices, are zero. Note that the dimension formulas for moduli spaces of curves in the symplectization $\mathbb{R} \times S^{*} Q$ and in the cotangent bundle $T^{*} Q$ discussed in Section 2 and 3 specialize to

$$
\begin{gathered}
\operatorname{dim} \mathcal{M}_{g}\left(\Gamma^{-}, \Gamma^{+}\right)=-\left(2-2 g-s^{+}-s^{-}\right) \\
\operatorname{dim} \mathcal{M}_{g, k}\left(T^{*} Q, Q ; \Gamma^{+}\right)=-\left(2-2 g-s^{+}-k\right) .
\end{gathered}
$$

Since the potential $\mathbf{F}$ is built from 0-dimensional moduli spaces of curves in $T^{*} Q$, we find that only genus zero curves with exactly two (positive) punctures contribute, so that

$$
\mathbf{F}=\frac{1}{\hbar} \sum_{i, j} e^{i j} p_{i} p_{j} .
$$

Using a maximum principle argument, one can prove that the only two-punctured spheres in $T^{*} Q$ are the trivial cylinders connecting $\gamma$ with $-\gamma$, so that $e^{i j}= \pm 1$ if the indices $i$ and $j$ correspond to one geodesic with its two possible orientations and $e^{i j}=0$ in all other cases. So, up to sign, we have completely determined the coefficients of $\mathbf{F}$. 
Similarly, since $\mathbf{H}$ is built from 1-dimensional moduli spaces of curves in the symplectization, we find that there are only four possible types of curves that contribute. In fact, $\left(g, s^{+}, s^{-}\right)$can only take the values

$$
(0,1,2),(0,2,1),(0,3,0) \text { and }(1,1,0)
$$

so that $\mathbf{H}$ has the form

$$
\mathbf{H}=\frac{1}{\hbar}\left(\sum_{i, j, k} a_{i j}^{k} q_{i} q_{j} p_{k}+\sum_{i, j, k} b_{i}^{j k} q_{i} p_{j} p_{k}+\sum_{i, j, k} c^{i j k} p_{i} p_{j} p_{k}+\hbar \sum_{i} d^{i} p_{i}\right) .
$$

Now recall that the chain map inducing the isomorphism of Theorem 7.1 is given by moduli spaces of holomorphic disks with one puncture and boundary on $Q \subset T^{*} Q$. Since the geodesics are absolutely length-minimizing in their homotopy classes, Lemma 7.2 implies that the only such half-cylinders are the trivial half-cylinders over the geodesics themselves. So the isomorphism is given by the map that sends each closed geodesic, viewed as a Reeb orbit, to its free homotopy class. In view of Theorem 7.1, and combined with the explicit knowledge of $\mathbf{F}$, this allows one to compute the coefficients $a_{i j}^{k}, b_{i}^{j k}$ and $c^{i j k}$ in $\mathbf{H}$ algebraically. For better illustration, here we instead present the pictures behind the algebra, which also allow us to determine the coefficients $d^{i}$.

To understand the counts $a_{i j}^{k}$ of spheres in the symplectization with 1 positive and 2 negative punctures, we consider the boundary of a 1-dimensional moduli space of spheres in $T^{*} Q$ with two boundary components on $Q$ and one punc-

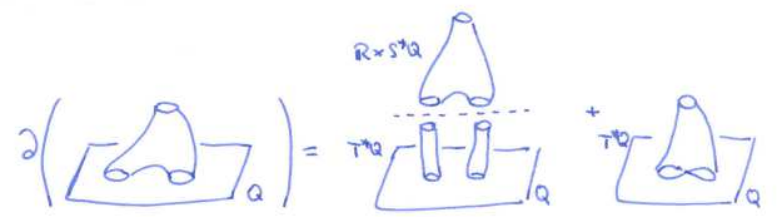

Figure 11: Codimension 1 phenomena in a moduli space of curves with two boundary components and one puncture

ture, given in Figure 11. We find that the $a_{i j}^{k}$ are (up to sign) identical to the coefficients of the string cobracket (represented by the second picture).

To understand the count $b_{i}^{j k}$ of spheres in the symplectization with 2 positive and 1 negative punctures, we consider the boundary of a 1-dimensional moduli space of spheres in $T^{*} Q$ with 1 boundary component on $Q$ and 2 punctures, given in Figure 12. Again this gives a linear equation, expressing the coefficients $b_{i}^{j k}$ in terms of the coefficients of the string bracket and the string cobracket.

To understand the count $c^{i j k}$ of spheres in the symplectization with 3 positive punctures, we consider the boundary of a 1-dimensional moduli space of spheres 

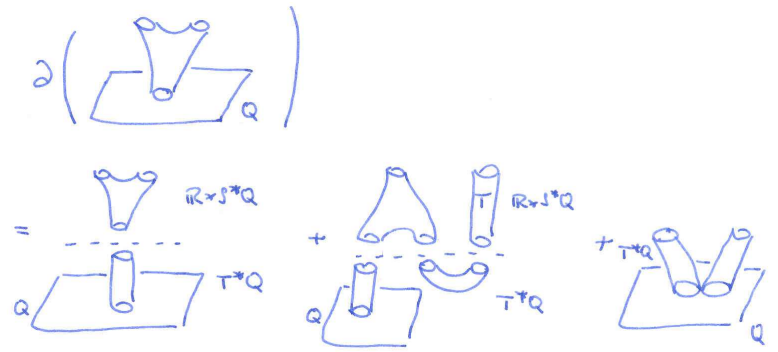

Figure 12: Codimension 1 phenomena in a moduli space of curves with one boundary component and two punctures

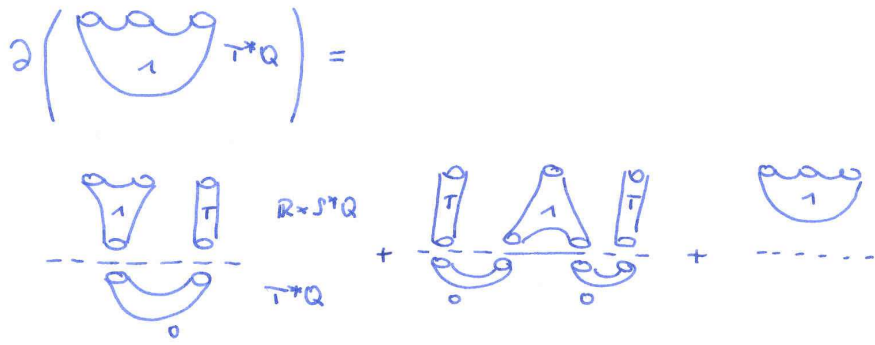

Figure 13: Codimension 1 phenomena in a moduli space of curves with three punctures and no boundary

in $T^{*} Q$ with 3 punctures (and no boundary), given in Figure 13, and conclude that the $c^{i j k}$ depend linearly on the $a_{i j}^{k}$ and the $b_{i}^{j k}$.

Finally, to understand the count $d^{i}$ of tori in the symplectization with 1 positive puncture, we consider the boundary of a 1-dimensional moduli space of tori in $T^{*} Q$ with 1 puncture (and no boundary), pictured in Figure 14, and conclude that the count is determined by the number of times a geodesic appears with both its orientations in the expression for the cobracket.

Taken together, we have sketched the proof of

Proposition 7.3. On an oriented closed surface $Q$ of genus $\geq 2$ with a metric of negative curvature, the coefficients of $\mathbf{H}$ are linear functions of the coefficients of the string bracket and cobracket. In particular, they are independent of the choice of the metric of negative curvature.

Note that each type of coefficient is non-zero for an appropriate choice of asymptotic geodesics. Specific examples are given in Figures 15-17. Here Figure 15 shows a configuration where the coefficient of $\frac{1}{\hbar} q_{\gamma} q_{\gamma^{\prime}} p_{\gamma^{\prime \prime}}$ is nonzero, and Fig- 


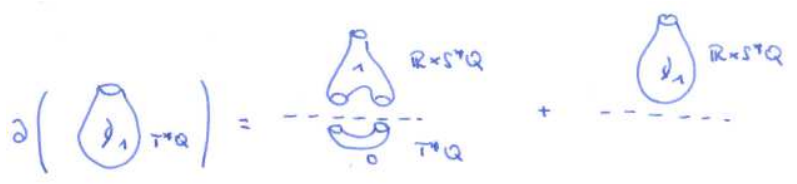

Figure 14: Codimension 1 phenomena in a moduli space of curves with genus 1 , one puncture and no boundary

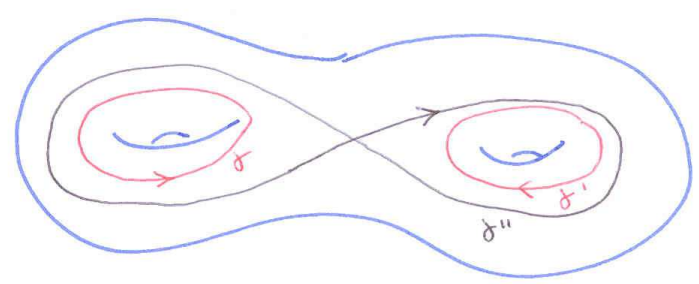

Figure 15: Nonvanishing string cobracket

ure 16 shows a configuration where the coefficient of $\frac{1}{\hbar} q_{\gamma} p_{\gamma^{\prime}} p_{\gamma^{\prime \prime}}$ is nonzero. In

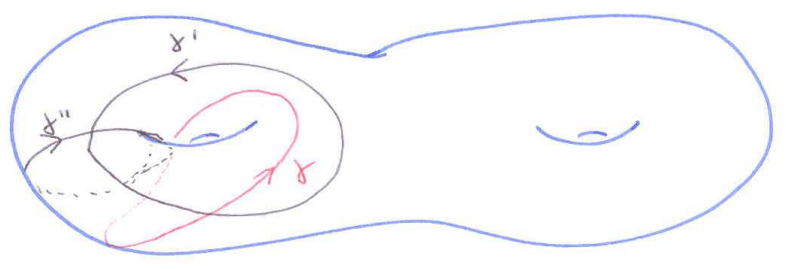

Figure 16: Nonvanishing string bracket

both cases, all other possible combinations of string brackets and cobrackets either vanish (Figure 15) or involve other homotopy classes (Figure 16), so that they also give examples for a nonzero coefficient of $\frac{1}{\hbar} p_{-\gamma} p_{-\gamma^{\prime}} p_{\gamma^{\prime \prime}}$ (Figure 15) and $\frac{1}{\hbar} p_{-\gamma} p_{\gamma^{\prime}} p_{\gamma^{\prime \prime}}$ (Figure 16), respectively.

Finally, Figure 17 gives an example for a nonzero coefficient of $p_{\gamma}$. Start with two curves $\gamma$ and $\gamma^{\prime}$ which differ only in their orientation (we have drawn them as parallel copies to improve readability of the picture). Then build a new curve $\gamma^{\prime \prime}$ by joining $\gamma$ and $\gamma^{\prime}$ as shown in green. The curve $\gamma^{\prime \prime}$ has three self-intersections, labelled by $x, y_{1}$ and $y_{2}$ in the picture. Applying the cobracket to $\gamma^{\prime \prime}$ means resolving each of them in turn. The resolution of $x$ clearly gives back $\gamma$ and 


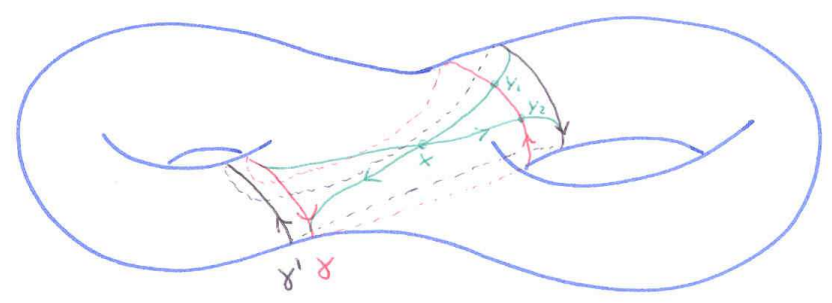

Figure 17: A curve appears with both orientations in a cobracket

$\gamma^{\prime}$, whereas one can check that the resolution of $y_{1}$ or $y_{2}$ gives curves in other homotopy classes.

\section{Technical remarks}

The details of the proof of Theorem 7.1, of course including the proof of Lemma 7.2, will be given in [9]. There we also give the precise form of the equations alluded to in Proposition 7.3, which depends on a detailed discussion of orientation conventions.

\section{General symplectic cobordisms}

In the previous sections we have considered only exact symplectic cobordisms. In this section we outline the generalization to non-exact cobordisms in terms of twisted $B V_{\infty}$-structures and discuss relations to Fukaya's work on Lagrangian embeddings.

In this section, let $\left(X^{2 n}, \omega\right)$ be a symplectic cobordism between contact manifolds $\left(Y^{ \pm}, \lambda^{ \pm}\right)$(i.e. $\left.\left.\omega\right|_{Y^{ \pm}}=d \lambda^{ \pm}\right)$with a compatible almost complex structure $J$. In addition, let $Q \subset X$ be a closed oriented relatively spin Lagrangian submanifold. We allow both $(X, \omega)$ and $Q$ to be non-exact. For simplicity, we assume that $H_{1}(X ; \mathbb{Z})$ has no torsion.

In the non-exact case we need to incorporate homology classes into the formalism, which is done as described in [16]: Fix a basis $A_{1}, \ldots, A_{N}$ for $H_{2}(X, Q)$ (modulo torsion) and introduce formal variables $z_{1}, \ldots, z_{N}$ with grading

$$
\left|z_{i}\right|:=-2 c_{1}\left(A_{i}\right),
$$

where $c_{1}$ denotes the first Chern class of the tangent bundle $(T X, J)$. Choose curves $b_{1}, \ldots, b_{M}$ representing a basis of $H_{1}(X ; \mathbb{Z})$ (which is free by assumption). For each closed Reeb orbit $\gamma$ of $Y^{+}$and $Y^{-}$we fix a capping surface connecting $\gamma$ to the corresponding linear combination of the $b_{i}$. These choices allow us 
to assign a class in $H_{2}(X, Q)$ to each punctured holomorphic curve in $X$ with boundary on $Q$.

Fix ordered collections of closed Reeb orbits $\Gamma^{ \pm}=\left(\gamma_{1}^{ \pm}, \ldots, \gamma_{s^{ \pm}}^{ \pm}\right)$, integers $g, k \geq$ 0 , and a relative homology class $d \in H_{2}(X, Q)$. Denote by

$$
\mathcal{M}_{g, k}^{d}\left(X, Q ; \Gamma^{-}, \Gamma^{+}\right)
$$

the moduli space of $J$-holomorphic curves of genus $g$ in $X$ with $s^{+}$positive and $s^{-}$negative punctures asymptotic to the $\gamma_{i}^{+}$resp. $\gamma_{j}^{-}$and with $k$ ordered boundary components on $Q$ representing the relative homology class $d$. This moduli space has expected dimension

$$
\begin{aligned}
& \operatorname{dim} \mathcal{M}_{g, k}^{d}\left(X, Q ; \Gamma^{-}, \Gamma^{+}\right) \\
& \quad=(n-3)\left(2-2 g-s^{+}-s^{-}-k\right)+\sum_{i} \mathrm{CZ}\left(\gamma_{i}^{+}\right)-\sum_{j} \mathrm{CZ}\left(\gamma_{j}^{-}\right)+\mu(d),
\end{aligned}
$$

where $\mu: H_{2}(X, Q) \rightarrow \mathbb{Z}$ denotes the Maslov class. Note that $\mu$ takes only even values because $Q$ is orientable. Simultaneous evaluation at the $k$ boundary components defines a map

$$
c_{g, k}^{d}\left(\Gamma^{-}, \Gamma^{+}\right): \mathcal{M}_{g, k}^{d}\left(X, Q ; \Gamma^{-}, \Gamma^{+}\right) \rightarrow \underbrace{\Sigma \times \ldots \times \Sigma}_{k}=: \Sigma^{k},
$$

which we again view as an element of the chain group $C_{*}\left(\Sigma^{k}\right.$, const $\left._{k}\right)$ defined in Section 3. As in the exact case, define the correlators

$$
\langle\underbrace{q, \ldots, q}_{s^{-}} ; \underbrace{p, \ldots, p}_{s^{+}}\rangle_{g, k}^{d}:=\sum_{\left|\Gamma^{ \pm}\right|=s^{ \pm}} c_{g, k}^{d}\left(\Gamma^{-}, \Gamma^{+}\right) q^{\Gamma^{-}} p^{\Gamma^{+}}
$$

and the potential $\mathbf{L}$ of the pair $(X, Q)$ by

$$
\mathbf{L}:=\frac{1}{\hbar} \sum_{g=0}^{\infty} \mathbf{L}_{g} \hbar^{g}
$$

where

$$
\mathbf{L}_{g}:=\sum_{s^{-}, s^{+}, k, d} \frac{1}{s^{-} ! s^{+} ! k !}\langle\underbrace{q, \ldots, q}_{s^{-}} ; \underbrace{p, \ldots, p}_{s^{+}}\rangle_{g, k}^{d} z^{d} .
$$

Thus $\mathbf{L}$ is a formal power series in $\hbar, z^{ \pm 1}$ and the $p_{\gamma}^{+}$, polynomial in the $q_{\gamma}^{-}$, with coefficients in the string algebra $\mathcal{C}$ of $Q$. Similarly, the Hamiltonians $\mathbf{H}^{ \pm}$ of $Y^{ \pm}$now include the variables $z$ encoding relative homology classes obtained from the embeddings $Y^{ \pm} \subset X$. With these modified definitions, the proof of Theorem 3.1 carries over verbatim to show

Theorem 8.1. The potential $\mathbf{L}$ of a (not necessarily exact) pair $(X, Q)$ satisfies the master equation

$$
\mathbf{D}_{\text {string }}\left(e^{\mathbf{L}}\right)=e^{\mathbf{L}} \overleftarrow{\mathbf{H}^{+}}-\overleftrightarrow{\mathbf{H}^{-}} e^{\mathbf{L}}
$$

where $\mathbf{D}_{\text {string }}=\partial+\Delta+\hbar \nabla$ is the $B V_{\infty}$-operator in string topology as defined in Section 6. 
Formally, equation (15) is identical to equation (8), but we will see that the interpretation is quite different. For simplicity, we assume from now on $Y^{-}=\varnothing$. Write

$$
\mathbf{L}=\mathbf{A}+\mathbf{B},
$$

where $\mathbf{A}:=\left.\mathbf{L}\right|_{p^{+}=0}$ encodes the holomorphic curves without punctures. The master equation specializes at $p^{+}=0$ to

$$
\mathbf{D}_{\text {string }}\left(e^{\mathbf{A}}\right)=0 \text {, }
$$

so $\mathbf{A}$ defines a Maurer-Cartan element in the $B V_{\infty}$-algebra $\left(\mathcal{C}[[z]], \mathbf{D}_{\text {string }}\right)$. As explained in Section 5, this leads to a twisted $B V_{\infty}$-operator

$$
\mathbf{D}_{\mathbf{A}}:=e^{-\mathbf{A}} \mathbf{D}_{\text {string }} e^{\mathbf{A}}
$$

on $\mathcal{C}[[z]]$. Now arguing as in Section 6 we find

Corollary 8.2. The map

$$
\mathbf{\Phi}: \mathcal{A}^{+}[[\hbar]] \rightarrow \mathcal{C}[[\hbar, z]], \quad g \mapsto e^{\overrightarrow{\mathbf{B}}} g
$$

is a $B V_{\infty}$-morphism from $\left(\mathcal{A}^{+}[[z]], \mathbf{D}_{\mathrm{SFT}}\right)$ to $\left(\mathcal{C}[[z]], \mathbf{D}_{\mathbf{A}}\right)$.

From here the theory in the general case is entirely parallel to the one in the exact case. The only difference is that non-exact symplectic cobordisms lead to $B V_{\infty^{-}}$ morphisms with respect to twisted $B V_{\infty}$-structures on the Lagrangian/negative side.

To conclude this section, let us explain how equation (16) is related to Fukaya's work [18]. First note that the $\mathrm{BV}_{\infty}$-operator $\mathbf{D}_{\text {string }}$ of string topology does not have higher order terms, so that according to (14) equation (16) is equivalent to

$$
\mathbf{D}_{\text {string }} \mathbf{A}+\frac{\hbar}{2}[\mathbf{A}, \mathbf{A}]_{\text {string }}=0 .
$$

Following a general principle known from SFT, there is a version of equation (16) for the part $\mathbf{a}:=\mathbf{A}_{0}$ of the generating function $\mathbf{A}$ corresponding to genus zero curves, which is obtained by considering the leading order term of the expansion of this equation in terms of $\hbar$. It takes the form

$$
(\partial+\Delta) \mathbf{a}+\frac{1}{2}[\mathbf{a}, \mathbf{a}]_{\text {string }}=0 .
$$

Specializing even further to the part $\mathbf{a}_{1}$ of $\mathbf{a}$ encoding genus zero curves with only one boundary component (i.e. holomorphic disks), we recover the equation

$$
\partial \mathbf{a}_{1}+\frac{1}{2}\left[\mathbf{a}_{1}, \mathbf{a}_{1}\right]_{\text {string }}=0,
$$

which was first observed by Fukaya [18]. In that paper, Fukaya introduces the idea of viewing moduli spaces of holomorphic disks as chains in string and loop 
space via evaluation at the boundary. He also outlines numerous applications of this circle of ideas to the topology of Lagrangian embeddings. These include the characterization of all aspherical irreducible closed oriented Lagrangian submanifolds in $\mathbb{C}^{3}$ (they must be a product of a circle with a surface) and a proof of a generalization of Audin's conjecture that each Lagrangian torus in $\mathbb{C}^{n}$ has minimal Maslov number two.

We remark that each of the potentials $\mathbf{A}, \mathbf{a}$ and $\mathbf{a}_{1}$ give rise to a twisted version of the corresponding differential in string topology. The resulting homologies are invariants of the Lagrangian embedding up to Hamiltonian isotopy.

We conclude this section with a discussion of the relation between holomorphic curves with punctures and curves with boundary. Consider again a closed Lagrangian submanifold $Q$ in a symplectic cobordism $X$ without negative end. We have seen that the part $\mathbf{A}=\left.\mathbf{L}\right|_{p^{+}=0}$ without positive punctures of the potential $\mathbf{L}$ yields a twisted $B V_{\infty}$-operator $\mathbf{D}_{\mathbf{A}}$ on the algebra $\mathcal{C}[[z]]$ of chains in string space. On the other hand, cutting out $Q$ we get a symplectic cobordism $X \backslash Q$ with negative end $S^{*} Q$. In this context, the discussion following Theorem 8.1 shows that the part $\mathbf{G}=\left.\mathbf{F}\right|_{p^{+}=0}$ without positive punctures of the potential $\mathbf{F}$ of $X \backslash Q$ yields a twisted $B V_{\infty}$-operator $\mathbf{D}_{\mathbf{G}}$ on the algebra $\mathcal{A}[[z]]$ of polynomials in $q_{\gamma}$ corresponding to the Reeb orbits on $S^{*} Q$. The following result states that these twisted operators are related by an isomorphism as in Theorem 7.1.

Theorem 8.3. In the situation above, there exists a $B V_{\infty}$-morphism between the twisted $B V_{\infty}$-algebras

$$
\Phi:\left(\mathcal{A}[[z]], \mathbf{D}_{\mathbf{G}}\right) \rightarrow\left(\mathcal{C}[[z]], \mathbf{D}_{\mathbf{A}}\right)
$$

which induces an isomorphism on homology.

Note that $\mathbf{G}$ counts holomorphic curves in $X \backslash Q$ with negative punctures asymptotic to $Q$, whereas A counts holomorphic curves in $X$ with boundary on $Q$ (both without positive punctures). So Theorem 8.3 gives a precise formulation of the principle that "holomorphic curves with punctures asymptotic to a Lagrangian submanifold $Q$ carry the same information as holomorphic curves with boundary on $Q$ ".

\section{Technical remarks}

Similarly to Theorem 3.1, Theorem 8.1 (and hence also Theorem 8.3) is conjectural at this point, with roughly the same analytic difficulties for the proof. As new phenomena, one now also has disk and sphere bubbling.

While the parity of the variables can be given precise meaning, the integer grading depends on the choice of capping surfaces used, and changing these capping surfaces shifts degrees by an appropriate (even) Maslov index, cf. [16].

To make the discussion more precise, one would also have to keep track of certain Novikov conditions as described in [16]. 


\section{Appendix A Towards relative SFT (joint with K. Mohnke)}

In this section we argue that string topology should play an essential role in relative symplectic field theory. This appendix is the result of joint work of the authors with K. Mohnke.

Let $\left(Y^{2 n-1}, \lambda\right)$ be a (not necessarily compact) contact manifold which is convex in the sense of [16]. For simplicity, we assume that there are no closed Reeb orbits. E.g. this is the case for $\mathbb{R}^{2 n-1}$ or, more generally, any 1-jet space with its canonical contact form. Let $\Lambda \subset Y$ be a compact Legendrian submanifold, which for simplicity we assume to have zero Maslov class.

Let us first recall the definition of relative contact homology as in $[7,16,14,15]$. A Reeb chord is a Reeb orbit starting and ending on $\Lambda$. We assume that all Reeb chords are nondegenerate. Let $J$ be a cylindrical almost complex structure on $\mathbb{R} \times Y$ adjusted to $\lambda$. For a cyclically ordered collection of Reeb chords $\Gamma=\left(\gamma_{1}, \ldots, \gamma_{s}\right)$ denote by $\mathcal{M}(\Gamma)$ the moduli space of $J$-holomorphic disks in $\mathbb{R} \times Y$ with boundary on $\mathbb{R} \times \Lambda$ and $s$ boundary punctures (in counterclockwise order) asymptotic to the $\gamma_{i}$. Here the punctures can be positive or negative in any order. The vanishing of the Maslov class allows us to assign to each Reeb chord $\gamma$ a Conley-Zehnder index $\mathrm{CZ}(\gamma) \in \mathbb{Z}$ such that the following dimension formula holds:

$$
\operatorname{dim} \mathcal{M}(\Gamma)=(n-3)\left(1-s^{+}\right)+\sum_{i \in I^{+}}\left(\mathrm{CZ}\left(\gamma_{i}\right)-1\right)-\sum_{j \in I^{-}}\left(\mathrm{CZ}\left(\gamma_{j}\right)-1\right) .
$$

Here $I^{ \pm} \subset\{1, \ldots, s\}$ are the sets of indices corresponding to positive resp. negative punctures and $s^{+}=\left|I^{+}\right|$. If this dimension is 1 denote by $n(\Gamma) \in \mathbb{Q}$ the algebraic count of points in $\mathcal{M}(\Gamma) / \mathbb{R}$.

Assign to each Reeb chord $\gamma$ a formal variable $q_{\gamma}$ of degree

$$
\left|q_{\gamma}\right|:=\mathrm{CZ}(\gamma)-1
$$

and let $\mathcal{A}$ be the free graded commutative algebra in the $q_{\gamma}$. Define a derivation $\partial: \mathcal{A} \rightarrow \mathcal{A}$ of degree -1 on generators by

$$
\partial q_{\gamma}:=\sum_{\gamma_{1}, \ldots, \gamma_{k}} n\left(\gamma_{1}, \ldots, \gamma_{k} \gamma\right) q_{\gamma_{1}} \cdots q_{\gamma_{k}},
$$

where the $\gamma$ corresponds to a positive puncture and $\gamma_{1}, \ldots, \gamma_{k}$ correspond to negative punctures. The following result is proved in [15] for 1-jet spaces and used to distinguish Legendrian submanifolds in all dimensions.

Theorem A.1. We have $\partial^{2}=0$ and the relative contact homology $H_{*}(\mathcal{A}, \partial)$ is an invariant of $\Lambda$ up to Legendrian isotopy.

Now let us turn to disks with more than one positive puncture. By analogy with absolute SFT, one would expect their counts to define operations on relative 
contact homology. For example, denote by $\mu: \mathcal{A} \otimes \mathcal{A} \rightarrow \mathcal{A}$ the operation obtained by counting rigid holomorphic disks in $\mathbb{R} \times Y$ with boundary on $\mathbb{R} \times \Lambda$ and two neighbouring positive punctures and an arbitrary number of negative punctures on the boundary. Figure 18 shows the codimension one degenerations of a 1-dimensional moduli space of disks with two positive boundary punctures.
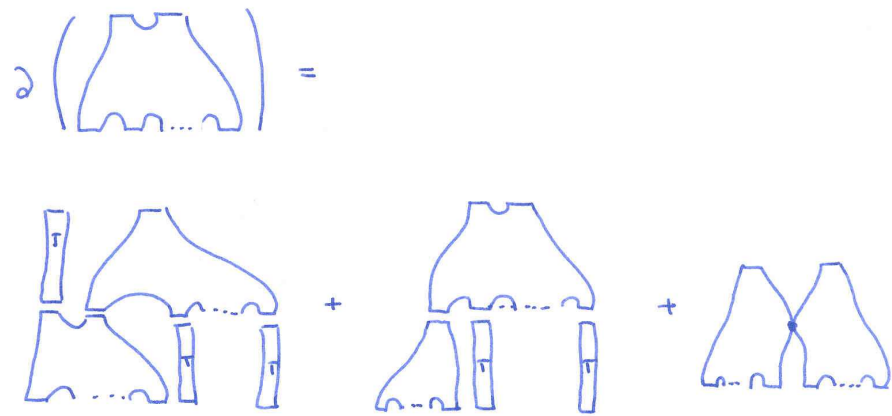

Figure 18: Codimension 1 phenomena in a moduli space of disks with two positive punctures

Without the last term, Figure 18 would show that $\mu \partial+\partial \mu=0$ and $\mu$ descends to $H_{*}(\mathcal{A}, \partial)$. However, this conclusion does not hold due to the presence of the last term and in general $\mu$ does not descend to an operation on relative contact homology. So how do we proceed from here?

One way is based on the observation that the last term is the string bracket (in $\Lambda$ ) of two moduli spaces with one positive boundary puncture. Moreover, since the string bracket has degree $3-n$, this term will for $n \geq 4$ involve moduli spaces of higher dimensions. Motivated by the previous sections, let us assemble the moduli spaces of all dimensions of holomorphic disks with arbitrary numbers of positive and negative boundary punctures into a Hamiltonian $\mathbf{h}$. Here the negative punctures correspond to the variables $q_{\gamma}$ and the positive punctures to variables $p_{\gamma}$ of degree

$$
\left|p_{\gamma}\right|:=n-2-\mathrm{CZ}(\gamma) .
$$

For each holomorphic disk, the evaluation at the boundary gives a broken closed string in $\Lambda$, which at the break points jumps from one end point of a Reeb chord to another. Thus $\mathbf{h}$ belongs to the space $\mathcal{P}$ of power series in the non-commuting variables $p_{\gamma}, q_{\gamma}$ (finite in the $q_{\gamma}$ ), with coefficients in the chains in the space of broken closed strings, modulo cyclic permutations acting diagonally, i.e. both on the chains and on the $p$ 's and $q$ 's. Consider the following operations on the space $\mathcal{P}$ :

- the singular boundary operator $\partial$; 
- the string bracket $[,]_{\text {string; }}$;

- the SFT bracket $[,]_{\mathrm{SFT}}$ given by deleting a pair $p_{\gamma}, q_{\gamma}$ and gluing the broken closed strings along the corresponding Reeb chord;

- the operation $\delta$ given by the action of a constant disk with one positive and one negative puncture at the same Reeb orbit $\gamma$ and with a fixed marked point on its boundary on the chains of broken closed strings by the loop-string-product as in Figure 19.
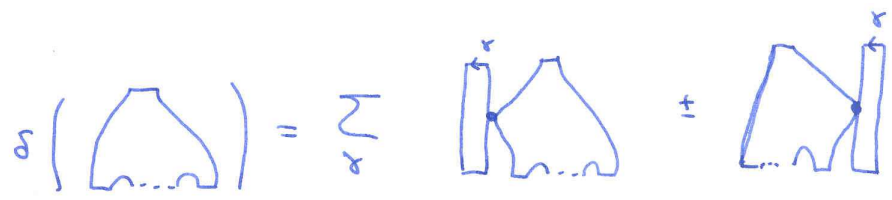

Figure 19: The operation $\delta$

Conjecture A.2. $[]:,=[,]_{\mathrm{SFT}}+[,]_{\text {string }}$ defines a Lie bracket of degree $3-n$ and $\partial+\delta$ defines an operator of square zero and degree -1 which is a derivation of this bracket. Moreover, the Hamiltonian $\mathbf{h}$ satisfies the equation

$$
(\partial+\delta) \mathbf{h}+\frac{1}{2}[\mathbf{h}, \mathbf{h}]=0 .
$$

The homology $H_{*}(\mathcal{P}, \partial+\delta+[\mathbf{h}, \cdot])$ is an invariant of $\Lambda$ up to Legendrian isotopy.

We see that, in contrast to the absolute case, in relative SFT string topology already enters in the definition of the theory.

For simplicity, we have formulated Conjecture A.2 for holomorphic disks and in the absence of closed Reeb orbits. For the full relative SFT one should consider arbitrary compact Riemann surfaces with boundary and positive and negative punctures in the interior as well as on the boundary. The algebraic formulation, analytical foundations, and applications of this theory are the subject of current research.

\section{Technical remarks}

In order to give Equation (18) rigorous meaning one must define the string operations on the chain level, with all the technical difficulties this entails. For the invariance proof, one also needs a discussion of the morphism induced by moduli spaces of curves in a cobordism and of homotopies between such morphisms. 


\section{References}

[1] A. Abbondandolo and M. Schwarz, On the Floer homology of cotangent bundles, Comm. Pure Appl. Math. 59, 254-316 (2006)

[2] F. Bourgeois, Y. Eliashberg, H. Hofer, K. Wysocki and E. Zehnder, Compactness results in symplectic field theory, Geom. \& Topol. 7 (2003), 799888

[3] F. Bourgeois and K. Mohnke, Coherent orientations in symplectic field theory, Math. Z. 248, 123-146 (2004).

[4] M. Chas, Combinatorial Lie bialgebras of curves on surfaces, Topology 43, 2004, 543-568

[5] M. Chas and D. Sullivan, String topology, preprint math.GT/9911159 (1999).

[6] M. Chas and D. Sullivan, Closed string operators in topology leading to Lie bialgebras and higher string algebra, math.GT/0212358.

[7] Y. Chekanov, Differential algebra of Legendrian links, Invent. Math. 150 (2002), no. 3, 441-483

[8] K. Cieliebak, K. Fukaya and J. Latschev, in preparation

[9] K. Cieliebak and J. Latschev, Symplectic field theory and string topology: the exact case, in preparation.

[10] K. Cieliebak and J. Latschev, Chain level string topology, in preparation.

[11] K. Cieliebak and K. Mohnke, Compactness for punctured holomorphic curves, Conference on Symplectic Topology, J. Symplectic Geom. 3 (2005), no. $4,589-654$

[12] K. Cieliebak and K. Mohnke, Symplectic hypersurfaces and transversality in Gromov-Witten theory, arXiv:math/0702887.

[13] K. Cieliebak, I. Mundet i Riera and D. Salamon, Equivariant moduli problems, branched manifolds, and the Euler class, Topology 42 (2003), no. 3, 641-700

[14] T. Ekholm, J. Etnyre and M. Sullivan, The contact homology of Legendrian submanifolds in $\mathbb{R}^{2 n+1}$, J. Diff. Geom. 71 (2005), no. 2, 177-305

[15] T. Ekholm, J. Etnyre and M. Sullivan, Legendrian contact homology in $P \times \mathbb{R}$, math $/ 0505451$

[16] Y. Eliashberg, A. Givental and H. Hofer, Introduction to symplectic field theory, GAFA 2000 Visions in Mathematics special volume, part II, 560673 
[17] Y. Eliashberg, Symplectic field theory and its applications, talk at the ICM 2006.

[18] K. Fukaya, Application of Floer homology of Lagrangian submanifolds to symplectic topology, in: Morse theoretic methods in nonlinear analysis and in symplectic topology, ed. by P. Biran, O. Cornea and F. Lalonde, $231-276$

[19] K. Fukaya, Y. G. Oh, H. Ohta and K. Ono, Lagrangian intersection Floer homology - anomaly and obstruction, preprint, 2000, revision 2006

[20] E. Getzler, Batalin-Vilkovisky algebras and two-dimensional topological field theories, Comm. Math. Phys. 159, 265-285 (1994).

[21] W. M. Goldman, Invariant functions on Lie groups and Hamiltonian flows of surface group representations, Invent. Math. 85 (1986), 263-302

[22] H. Hofer, K. Wysocki and E. Zehnder, Polyfolds and Fredholm Theory $I-V$, in preparation.

[23] O. Kravchenko, Deformations of Batalin-Vilkovisky algebras, Poisson geometry (Warsaw, 1998), 131-139, Banach Center Publ., 51, Polish Acad. Sci., Warsaw, 2000

[24] H.-B. Rademacher, On the average indices of closed geodesics, J. Diff. Geom. 29, 65-83 (1989).

[25] D. Salamon and J. Weber, Floer homology and the heat flow, Geom. Funct. Anal. 16 (2006), no. 5, 1050-1138

[26] D. Tamarkin and B. Tsygan, Noncommutative differential calculus, homotopy $B V$ algebras and formality conjectures, Methods Funct. Anal. Topology 6 (2000), no. 2, 85-100

[27] V. G. Turaev, Skein quantization of Poisson algebras of loops on surfaces, Ann. Sci. Ecole Norm. Sup. (4) 24 (1991), 635-704

[28] C. Viterbo, Functors and computations in Floer homology with applications, preprint 1998. 